\title{
Savoirs vs pratiques en histoire : effets et conditions didactiques de dépassement d'un inconscient scolaire disciplinaire
}

Knowledge vs practices in history: impacts and didactics conditions to overcome an academic disciplinary unconscious

Sylvain Doussot

URL : http://journals.openedition.org/educationdidactique/2747

DOI : 10.4000/educationdidactique. 2747

ISSN : $2111-4838$

\section{Éditeur}

Presses universitaires de Rennes

\section{Édition imprimée}

Date de publication : 6 décembre 2017

Pagination : 103-130

ISBN : 978-2-7535-7318-5

ISSN : $1956-3485$

\section{Référence électronique}

Sylvain Doussot, «Savoirs vs pratiques en histoire : effets et conditions didactiques de dépassement d'un inconscient scolaire disciplinaire », Éducation et didactique [En ligne], 11-2 | 2017, mis en ligne le 06 décembre 2019, consulté le 19 avril 2019. URL : http://journals.openedition.org/ educationdidactique/2747 ; DOI : 10.4000/educationdidactique.2747 


\title{
SAVOIRS VS PRATIQUES EN HISTOIRE : EFFETS ET CONDITIONS DIDACTIQUES DE DÉPASSEMENT D'UN INCONSCIENT SCOLAIRE DISCIPLINAIRE
}

\author{
Sylvain Doussot \\ ESPE et CREN (EA 2661), université de Nantes
}

\begin{abstract}
Linconscient scolaire (Bourdieu) peut se spécifier dans l'habitus professionnel des enseignants d'une discipline, et sa connaissance est un enjeu essentiel de formation et d'analyse de l'apprentissage. Pour l'histoire, il s'incarne en particulier dans la dichotomie des contenus de savoir et des pratiques pédagogiques, dont les multiples manifestations, parfois contradictoires, produisent des effets de domination qui contraignent les enseignants comme les élèves. Parmi ces effets, l'absence de reconnaissance d'une approche scientifique des phénomènes d'enseignement et d'apprentissage, que visent les didactiques des disciplines, a des conséquences sur les conditions de possibilité du dépassement de cette dichotomie par les enseignants, les élèves et les chercheurs. L'article utilise des outils socioépistémologiques pour mettre en perspective des recherches didactiques qui tiennent ensemble structures cognitives et structures institutionnelles, de la communauté scientifique à la classe, pour identifier les articulations possibles entre apprentissage, enseignement, formation à l'enseignement et recherche, et proposer l'hypothèse d'une formation par exemple exemplaire (au sens de Kuhn) de construction et résolution de problèmes didactiques.
\end{abstract}

Mots-clés : inconscient scolaire, didactique, histoire, enseignement, problématisation.

Knowledge vs practices in history: impacts and didactics conditions to overcome an academic disciplinary unconscious

The academic unconscious (Bourdieu) can be specified through the professional habitus of the teachers of a discipline. Such a knowledge is a key issue for training and researches in learning. The History academic unconscious can be seen as the dichotomy of knowledge and teaching practices which expressions, sometimes contradictious, produce domination effects that command teachers as well as pupils. Among those effects the lack of acknowledgement of a possible scientific approach of teaching/learning phenomenon (didactics as a science) has consequences on the possibility for teachers, pupils and researchers to overcome this dichotomy. The paper is based on socio-epistemologic tools to put into perspective didactics researches that hold together cognitive structures and institutional structures from the scientific community to the classroom, in order to identify possible connections between learning, teaching, teachers training and research, and to consider training through exemplars (Kuhn) of problem building and solving.

Keywords: academic unconscious, didactics, history, teaching, problematization. 


\section{INTRODUCTION}

La mise au jour de l'inconscient scolaire (Bourdieu, 2000b) peut constituer un levier important pour appréhender les conditions du développement des acteurs de l'école (le développement professionnel pour les enseignants et les chercheurs, et l'apprentissage pour les élèves). Si « l'analyse de cet inconscient [qui] doit s'appliquer en priorité à la relation, jusqu'à ce jour à peine explorée, entre les structures institutionnelles (l'histoire des disciplines par exemple) et les structures cognitives ou, plus précisément, leur objectivation dans des savoirs et des connaissances » (Bourdieu, 2000b, p. 4), l'étude de l'enseignement-apprentissage d'une discipline telle que l'histoire - prise ici comme objet - peut constituer une occasion pour en éclairer des aspects fondamentaux. Plus encore, l'étude de la formation didactique à l'enseignement-apprentissage d'une discipline peut se révéler une double occasion d'exploration de certaines dimensions de l'inconscient scolaire. En effet, si le regard didactique vise à saisir les conditions de possibilité de l'accès des élèves à des savoirs scientifiques, il porte simultanément un enjeu d'analyse de la pratique enseignante comme une condition essentielle de cet accès.

Dans cette configuration, différentes strates de structures institutionnelles sont en jeu : la discipline scolaire, entendue comme le résultat d'une construction historique qui autonomise progressivement les contenus, pratiques et finalités scolaires de leur référence savante (Chervel, 1988) ; les cursus de formation initiale des enseignants de la discipline (et en particulier dans leur forme actuelle en France, les masters universitaires professionnalisant aux métiers de l'enseignement, de l'éducation et de la formation, spécifiés par une mention second degré et des parcours disciplinaires) ; les disciplines universitaires (comme l'histoire) qui participent à cette formation; ou encore l'organisation du système scolaire (généralement appréhendable à une échelle nationale) qui détermine les relations professionnelles et hiérarchise les parcours d'élèves. Or, « on peut supposer que les grandes divisions (et hiérarchies) scolaires, en disciplines, en formes d'exercices (cours magistral vs séminaire par exemple), tendent à se reproduire dans des principes de vision et de division spécifiques, eux-mêmes enveloppés dans des principes plus généraux qui les soutiennent »(Bourdieu, 2000b, p. 4). Ainsi, parmi les dichotomies impensées de l'histoire scolaire, celle qui sépare les contenus de savoir des pratiques pédagogiques est certainement l'une des plus profondément inculquée par le système d'enseignement (primaire, secondaire et supérieur) dans lequel les enseignants (et les chercheurs) sont immergés depuis leur expérience d'élèves.

Parmi les sciences sociales, les didactiques disciplinaires visent précisément à mettre en question ce type de division portée par les acteurs sociaux. La didactique de l'histoire, en particulier à travers le cadre d'analyse de la discipline scolaire (issu de travaux en histoire de l'enseignement ${ }^{1}$ ) a développé depuis quelques décennies des travaux descriptifs et critiques sur l'articulation des contenus, des pratiques et des finalités de la discipline scolaire correspondante (Lautier \& Allieu-Mary, 2008). Certains de ces travaux décrivent en particulier la puissance de l'inertie de cette discipline scolaire (Tutiaux-Guillon, 2008) et sa manière propre et indépendante de lier contenus et pratiques par rapport à la discipline universitaire de référence. Le recours à une approche sociologique générale - inscrite dans une perspective unifiée des sciences sociales ${ }^{2}$ - et à la notion d'inconscient scolaire en particulier, rend possible l'élargissement de la focale d'analyse de cette inertie et des conditions de possibilité d'en sortir en rapportant notamment l'articulation des contenus de savoir et des pratiques pédagogiques de la discipline scolaire à celle de la discipline universitaire. L'enjeu de cette comparaison réside notamment dans une focalisation sur les enseignants du secondaire dont l'expérience - davantage encore pour les débutants - est mixte, à la fois marquée par leur passage par l'enseignement scolaire de l'histoire et par leur formation universitaire en histoire. Il s'agit donc dans cette étude de faire un détour par des débats épistémologiques et pratiques ancrés dans les sciences sociales (sociologie, histoire et didactique essentiellement) pour mettre en perspective l'approche didactique de l'enseignement de l'histoire autour de la notion de discipline ${ }^{3}$ pensée en tension entre les nécessités de la communication pédagogique et l'exigence d'innovation $^{4}$ (Fabiani, 2006, p. 13).

\section{UNE DICHOTOMIE QUI S'ÉTEND BIEN AU-DELÀ DE L'ÉCOLE}

Déjà soulignée par Durkheim (1938) et Dewey (1990), la dichotomie des savoirs et des pratiques 
pédagogiques est au cœur des débats sur l'École en France dans les années 1970-1980 que symbolise l'ouvrage polémique de Milner (1984), qui dénonce le "roman pédagogique " en des termes "manichéens ${ }^{5} »$ : «Question tabou : la thèse familière des spécialistes de l'éducation et des réformateurs qui, explicitement ou implicitement, s'en inspirent, c'est que les contenus n'importent pas. Seule importe la forme, c'est-à-dire les méthodes pédagogiques : celles-ci seront d'autant plus pures que les contenus seront d'autant plus pauvres [...]. La Réforme s'est ornée au fil des ans de multiples patronymes (Fouchet, Fontanet, Haby, Legrand, etc.) ; un seul en vérité lui convient et c'est Dupont-la-Joie » (Milner, 1984, p. 140). La dichotomie contenus / pédagogie devient un objet de discussion et de travail lorsque se fondent les approches didactiques des disciplines, dans les années 1970 pour la France, et dans les années 1980 pour l'Amérique du nord ${ }^{6}$. Elle est toujours vivace dans les débats politiques autour de la place des disciplines dès que sont en jeu de nouveaux programmes et des demandes sociales nouvelles, comme l'illustre l'intervention du président de l'APHG (Association des Professeurs d'Histoire-Géographie) dans une lettre à la ministre de l'Éducation à l'occasion du récent renouvellement de programmes : "L'Association des professeurs d'Histoire et de Géographie (APHG) vous demande solennellement, en ces moments cruciaux pour l'avenir de la République, que le Conseil supérieur des programmes (CSP) ne fasse pas disparaître ou diminuer les savoirs au profit des compétences, de façon que tous les enfants de France puissent avoir les mêmes repères solides et la culture nécessaire à la conjuration des peurs et des haines. Elle vous demande aussi de garantir toute leur place aux savoirs dans les procédures de recrutement des futurs enseignants ${ }^{7} »$.

\section{Une dichotomie au cour de la recherche} didactique

La mise en discussion de cette dichotomie par les recherches en didactique repose sur l'étude des conditions de la transposition (dans la structure institutionnelle que constitue la " classe d'histoire ») des rapports entre les savoirs scientifiques objectivés dans des corpus de textes, et les pratiques instrumentées spécifiques à la communauté des historiens qui les produisent. Traditionnellement, si les contenus sont normés par différents instruments institutionnels - des textes savants de référence aux instructions officielles qui obligent les enseignants, en passant par une littérature intermédiaire de recommandations, de ressources et d'accompagnement, des manuels, et la formation - les pratiques restent quant à elles très peu formalisées et sont institutionnellement renvoyées à la liberté pédagogique des enseignants. Cette faible formalisation de la pédagogie contraste avec les enjeux qu'elle porte pourtant, pour les enseignants et au-delà dans la société, comme l'illustrent régulièrement des polémiques publiques telles celles sur les méthodes d'enseignement de la lecture ou les modalités d'enseignement de l'histoire de la Shoah. Ces enjeux percent régulièrement dans la vie des classes, des enseignants et des autres acteurs de l'institution, comme lors d'une inspection, où l'absence de référentiel explicite fait reposer l'évaluation des pratiques pédagogiques sur des traditions qui peuvent être différemment connues et interprétées.

La formation des enseignants, de ce point de vue, fait exception parce qu'elle constitue l'un des rares lieux de questionnement explicite de la pédagogie, parce qu'il y est nécessaire de mettre en textes (écrits ou oraux) les pratiques. C'est d'ailleurs dans le giron des institutions de formation des enseignants que se sont développées les recherches en didactique des disciplines, face à la nécessité perçue par les acteurs de cette formation d'en raisonner l'enseignement (Johsua \& Lahire, 1999), et notamment par le questionnement des rapports que peuvent entretenir savoirs et pratiques pédagogiques. Cette double caractéristique - lieu d'explicitation des discours sur les contenus et les pratiques, et lieu de développement d'une recherche institutionnalisée en didactique - conduit à envisager les situations de formation comme particulièrement propices à l'appréhension de cet allant de soi que constitue l'inconscient scolaire lié à l'enseignement de la discipline historique.

\section{LA DICHOTOMIE SAVOIRS / PÉDAGOGIE CHEZ LES ÉLÈVES ET LES PROFESSEURS}

On peut entamer la description des modalités variées des effets de la dichotomie savoirs / pédagogie du point de vue des élèves. Dans les classes, les savoirs sont mis en scène dans des activités propres 
à chaque discipline. Pour l'histoire, ces activités sont majoritairement constituées d'études de documents dont les enjeux sont nettement séparés des contenus, proposés quant à eux en général sous forme de textes descriptifs et explicatifs qui concluent les séances de travail. La mise en activité des élèves par l'étude de documents dits « historiques » s'est installée progressivement dans les habitudes professorales au cours du $\mathrm{XX}^{\mathrm{e}}$ siècle (Garcia \& Leduc, 2003), et des enquêtes quantitatives (Braxmeyer, 2007) indiquent qu'elles constituent une composante normale du cours d'histoire dans le secondaire. Le clivage entre le temps d'activité des élèves et la narration du professeur qui structure et conclut la séance est une des caractéristiques de l'enseignement de l'histoire que formulent les élèves, et dont rend compte l'analyse didactique.

Les pratiques attendues des élèves peuvent faire l'objet de mises en texte sous forme de méthodologies, souvent oralement lors des phases de correction, ou bien de manière plus formelle (et souvent écrite) dans des dispositifs institutionnels comme l'accompagnement personnalisé. Les enseignants ont mené loin le travail de catégorisation qui fait des méthodes (d'étude de documents, d'écriture et d'argumentation) la dimension enseignable des activités attendues des élèves - notamment lors des examens -, mais surtout qui norme les pratiques attendues en une compétence spécifiquement scolaire acquise par les professeurs au fil de leur expérience d'élève et d'étudiants dans un environnement institutionnel de plus en plus isolé d'autres disciplines (y compris des sciences sociales) et de la recherche.

Du côté des enseignants comme du côté des élèves, la dichotomie des contenus et des pratiques s'actualise ainsi dans une différenciation marquée entre des contenus historiques écrits et légitimés, et des pratiques pédagogiques (pour les enseignants) ou d'étude de documents (pour les élèves) généralement oralisées, ce qui n'est pas sans conséquence sur les conditions de possibilité d'une réflexion sur les pratiques.

La dualité écrit / oral se retrouve au niveau de l'historiographie elle-même, et renforce la dichotomie des savoirs et de la pédagogie, comme l'analyse Françoise Wacquet à travers l'intérêt porté par les historiens aux écrits de recherche au détriment de l'oral d'enseignement :

« saisi dans sa dimension enseignante, orale, [l'exercice du métier d'historien] n'a guère donné lieu à réflexion.
On retrouve ici la méfiance, voire l'hostilité manifestée par l'Université à l'égard de la pédagogie, opinion forte ancienne puisque Durkheim la qualifiait déjà dans le cours qu'il donna à la Sorbonne en 1904-1905 de "vieux préjugé". [...] Ce qui ne veut pas dire que l'on ne soit point intéressé au métier. Mais dans l'Université - et pour s'en tenir ici à l'histoire -, la réflexion sur "le métier d'historien" a presque entièrement porté sur la fabrique de l'histoire, sur "comment on écrit l'histoire", non sur comment on la dit $»$ (Waquet, 2000, p. 42).

L'histoire de l'enseignement conduit à un constat similaire si l'on suit l'introduction d'Annie Bruter à un dossier sur l'histoire de l'enseignement de l'histoire : " Tout se passe alors comme s'il n'y avait et s'il ne pouvait y avoir qu'une seule manière de l'enseigner, comme si cette discipline scolaire était ellemême sans histoire hors celle de ses contenus » (Bruter, 2007, p. 5). Dès lors, la réflexion sur les pratiques est mal engagée. D'abord du fait de l'absence d'apprentissage des «technologies de l'intellect » (Goody, 1979) que permet l'écrit mais pas l'oral, mais aussi du fait de la négation du rôle de médiateur social que peuvent jouer ces outils qui, sortant l'énoncé de son contexte, conduisent (ou non) à des échanges sur cette recontextualisation; les effets didactiques de ces instruments graphiques sont nettement différenciateurs dans la problématisation en histoire scolaire ${ }^{8}$.

Pour les enseignants, notamment débutants, les fiches de préparation reflètent aussi une centration sur les contenus, inscrits dans des cadres censés soutenir une organisation de l'activité pédagogique qui se limite à des catégorisations standardisées définissant en général des supports et une répartition du travail, le tout dans une chronologie précise, mais qui vont rarement jusqu'à faire état des enjeux de savoir et d'apprentissage qui diraient comment lier contenus visés et activités prévues. La généricité des catégories d'action utilisées et la rigidité formelle de ce type de support de la pratique pédagogique donnent aux contenus la prééminence sur les activités planifiées pour les élèves, et excluent d'emblée la possibilité de prendre en compte de manière réfléchie les incertitudes de la situation pratique. En somme, ces modalités d'organisation de la pratique d'enseignement supposent que les activités des élèves se limitent à l'application de règles, parfois inscrites dans des fiches de méthodologies; la responsabilité du passage des règles de méthode à la production des 
textes repose de ce fait entièrement sur les élèves. Finalement, dans le travail pédagogique des enseignants comme dans le travail scolaire des élèves, l'intégration effective des savoirs et de la pratique repose sur un travail invisible, individuel et indiscuté, qui suppose des choix permanents mais difficiles à mettre en question et en discussion, qui sont masqués par l'évidence d'une mise en texte des règles d'action sur le savoir en guise de pensée de la pratique. La forme scolaire d'une discipline comme l'histoire fait ainsi alterner des explications et des entraînements ${ }^{9}$ et maintient close la boîte noire de la pratique comme application de règles : l'enseignement de l'histoire est « coulé dans la forme scolaire » (Tutiaux-Guillon, 2008, p. 126).

Une revue des textes para-officiels que constituent les nombreuses « ressources » de "mise en ouvre des programmes ", à la disposition des enseignants sur le site dédié eduscol, manifeste l'écart entre une mise en texte des pratiques pédagogiques sous forme de règles et leur mise en application, en montrant en creux la difficulté de la synthèse qui reste de la charge individuelle des enseignants. Cela se voit dans la répétition de la nécessité d'une progression, sans que celle-ci ne soit jamais explicitée au-delà de la formulation des extrêmes entre lesquels elle doit se situer (par exemple, conduire de manière autonome une mise à distance critique vs écouter le professeur se livrer à cette opération), mais qui passerait par « un apprentissage régulier et progressif », et non de simples pratiques « rituelles» (nous soulignons les éléments de ce type par l'italique dans l'extrait suivant) :

"La capacité "porter un regard critique" est en jeu dès l'apprentissage de la présentation d'un document. Cette présentation doit, autant que possible, être porteuse de sens, dépasser une pratique un peu "rituelle" où l'on énumère des éléments (auteur, date, nature...) sans en tirer le moindre éclairage en termes de contextualisation du document, d'intentionnalité de son auteur, de public visé donc de portée potentielle de ce document. On ne peut pas attendre d'un jeune élève qu'il conduise cette mise à distance critique en autonomie, mais il doit prendre l'habitude de cette exigence, d'abord en écoutant son professeur se livrer à cette opération puis en étant guidé pour la réaliser par luimême. Le travail sur document doit faire l'objet d'un apprentissage régulier et progressif dans tous ses aspects - identification, contextualisation, prélèvement d'informations, confrontation avec d'autres sources, mise à distance critique - en adoptant une démarche qui permette à l'élève de passer d'un guidage fort à une activité intellectuelle un peu plus autonome ${ }^{10}$.»
Comme l'ont montré les études descriptives de la discipline scolaire dans les travaux didactiques depuis un quart de siècle, une inertie puissante du fonctionnement de l'histoire scolaire (Tutiaux-Guillon, 2008) maintient une séparation forte des savoirs et des pratiques qui indique que la conjonction des structures institutionnelles et des structures cognitives de l'enseignement de l'histoire est suffisamment consistante et généralisée pour ne pas être minorée par les facilités d'une explication externe, qui tendrait à renvoyer l'origine des pratiques actuelles et leurs effets à des choix politiques qui viseraient volontairement une instrumentalisation des savoirs et des disciplines. Si la rhétorique de l'utilitarisme prend une place croissante dans les discours publics en faisant de l'enseignement et de l'apprentissage d'une discipline comme l'histoire le lieu du développement de capacités et de compétences directement utiles à l'employabilité et au développement personnel dans une perspective de gestion de soi ${ }^{11}$, cette évolution s'inscrit dans un environnement institutionnel et cognitif disposé à les accueillir. En outre, la nouveauté des mots pour le dire (comme celui aujourd'hui dominant de compétences) qui passent rapidement des instances politiques internationales à celles du ministère de l'Éducation, et dans les textes règlementaires, ne doit pas faire oublier qu'ils correspondent à des préoccupations et donc des catégories anciennes des enseignants et des élèves, sous d'autres termes (savoir-faire, méthodes, etc.). Rejeter la faute, ou tout au moins la cause, sur des facteurs externes empêcherait de questionner l'assimilation des compétences à des savoirfaire techniques, c'est-à-dire de les penser dans leurs rapports aux situations de manipulation du savoir, par définition toujours singulières et incertaines.

\section{LES EFFETS DE DOMINATION DE LA DICHOTOMIE}

Les différents niveaux d'influence de la dichotomie se conjuguent pour produire des effets de domination $^{12}$ que l'on peut décrire chez les élèves, les enseignants et les chercheurs.

\section{Élèves dominés}

Le développement d'une vulgate constructiviste dans l'enseignement de l'histoire, en opposition avec le cours magistral, met en tension chez les ensei- 
gnants leurs conceptions du savoir et celles de l'apprentissage. Si le savoir est pour eux incarné par les textes, faire la classe renvoie d'abord à une mise en activité des élèves qui induit une focalisation sur le « comment faire ». Ce dualisme est soutenu institutionnellement comme le montre le déploiement parallèle des programmes (qui listent des contenus) et, depuis 2005, des " compétences » (dans le cadre de ce qu'on appelle « le socle commun ${ }^{13} »$ ). L'étude des pratiques ordinaires rend compte des effets de domination de cette dichotomie, qui relève tant de la structure institutionnelle de la classe que de sa structure cognitive. On ne la voit jamais aussi bien que dans la volonté affichée par les enseignants d'histoire-géographie de faire construire la trace écrite (c'est-à-dire le texte du savoir comme pur contenu) par les élèves. Pour ces derniers, le rapport entre le travail fourni et les textes à apprendre reste souvent obscur et du ressort du professeur, que ces élèves soient performants ou non selon les critères scolaires. De l'avis des professeurs (Lautier, 1997), rares sont les élèves en mesure de produire des textes cohérents et complets, et il est difficile de construire des études de documents adaptées, accessibles aux élèves et qui permettent de "faire construire » le savoir visé. Ce hiatus entre texte du savoir et pratiques de classe apparaît en creux dans les textes d'accompagnement produits par le Ministère qui traitent régulièrement de ce problème, à la fois pour en montrer les limites fortes (ne pas imaginer tout faire découvrir par les documents), et en proposer des évolutions (par exemple les études de cas ou études ciblées qui renouvellent la dichotomie : si l'étude de documents centrés sur une situation est du ressort des élèves, sa mise en perspective est du ressort du professeur ${ }^{14}$ ). Ces pratiques ordinaires et ces textes institutionnels manifestent un grand silence sur les enjeux de ces pratiques et leur relation avec la validité des savoirs, et illustrent un partage du travail et des responsabilités pour le moins paradoxal : le travail des élèves est un travail productif - ils produisent des réponses aux questions - alors que le travail de l'enseignant est constructif - il tire des réponses disparates à des questions parcellaires, des explications générales qui font référence, notamment lors des évaluations.

D'un point de vue didactique, le fossé perçu par les élèves entre activités sur documents et textes de synthèse se double d'un écart important entre activités de classe et pratiques historiennes : si les historiens travaillent bien sur des sources documentaires, ce travail n'a que peu à voir avec celui demandé aux élèves, qui se limite le plus souvent à faire sélectionner des informations et à reproduire un savoir censé être acquis ${ }^{15}$. Malgré les évolutions indéniables de la discipline scolaire au cours du temps, les rapports entre activités d'enseignement et activités d'apprentissage restent stables. Un texte de savoir est visé, qui doit expliquer le passé ; la (re)construction de l'explication constitue l'objectif de savoir-faire, que l'enseignant décline sous forme de méthode, elle-même mise en texte. Dans cette perspective, le contenu est considéré comme unique et fruit de l'application d'une méthode, ce qui renvoie l'échec des élèves à un défaut de respect des règles. Le texte est réifié, tandis que la singularité intrinsèque de la pratique - chaque étude de documents est singularisée, précisément et intrinsèquement, par ses documents : l'histoire est une science du singulier - n'est pas questionnée : les « compétences» scolaires réduisent la capacité d'agir sur les savoirs (documents, leçons précédentes...) à l'application de techniques, ignorant l'enjeu central que peut recouvrir l'idée de compétence lorsqu'elle est couplée à celle de situation (Rey, Carette, Defrance \& Kahn, 2006; Rey, 2011), qui consiste à savoir dans quelle situation appliquer quelle technique (ou dit autrement, la compétence est celle de l'analyse des situations d'étude). Dans ce cadre dominant, l'élève est laissé seul face à l'application de règles de méthode sur des contenus qui ne peut suffire pour agir pertinemment.

\section{Enseignants dominés}

La dichotomie des contenus et de la pédagogie constitue également un support idéologique favorable au maintien d'une position dominée des enseignants dès qu'ils sortent de leurs classes. Ils sont d'abord dominés par les producteurs de savoirs, eux qui ne sont que transmetteurs ${ }^{16}$, au sein d'un partage du travail qui ne se manifeste jamais autant que dans des situations où les deux catégories se côtoient. C'est l'intérêt de l'étude que propose Seixas (1999) d'une situation de formation dans laquelle des historiens, des sociologues et des anthropologues sont invités à participer à un séminaire de formation continue d'enseignants scolaires, qui décrit les déterminants de la « remarquable endurance [tremendous staying power] » de la dichotomie (Seixas, 1999, p. 318). Une telle étude de cas permet en effet de saisir les 
manifestations de cette domination pour mieux en comprendre les conditions de possibilité ${ }^{17}$. Sa pertinence réside dans la remise en cause potentielle qu'elle porte de l'inconscient scolaire professoral $\mathrm{du}$ formateur et du chercheur, toujours très proche par position et trajectoire, de l'inconscient scolaire étudié.

Parmi les déterminants de la persistance de la dichotomie, les statuts des uns et des autres légitiment la dépendance des enseignants envers les chercheurs et leurs textes. Derrière les statuts, ce sont les appartenances institutionnelles qui ont des effets épistémologiques et politiques forts. Dans la situation d'interaction, les historiens adaptent volontairement leurs discours aux enseignants considérés comme n'étant pas au fait des questionnements épistémologiques, en dogmatisant les résultats de leurs recherches : " c'était dans ce contexte - dans lequel ils [les historiens] communiquaient avec ceux qui sont en dehors de la communauté scientifique, et des enseignants scolaires en particulier - que leur savoir s'était réifié en contenu »(Seixas, 1999, p. 322).

On peut aisément rattacher l'acceptation réciproque de cette domination aux parcours de formation des enseignants du second degré qui, en France comme en Amérique du nord, s'organise autour de la dichotomie des savoirs disciplinaires et de la pédagogie, en donnant la part belle aux premiers, dans une relation de dépendance forte qui soumet les étudiants à des spécialistes de leur domaine sans réellement les faire accéder à la production et aux conditions de production des savoirs. Dans le contexte français, une petite proportion d'étudiants reçus aux concours de l'enseignement engagent ou ont engagé des recherches en histoire. Ce rapport déséquilibré se signale également dans nombre de réflexions et d'essais sur l'enseignement de l'histoire dans lesquels l'idéal difficilement atteignable serait que les enseignants soient enseignants et chercheurs ${ }^{18}$.

Les débats récents mais récurrents autour de la dimension professionnelle des épreuves des concours de recrutement des professeurs d'histoire-géographie laissent transparaître la résistance de la forme dominante (composition et commentaire de documents) contre les dimensions didactiques introduites par les textes réglementaires ${ }^{19}$. Ces débats peuvent en particulier être appréhendés par le biais de revues telle que Le Débat, dont deux numéros rapprochés ${ }^{20}$ se penchent sur la question de l'enseignement de l'histoire, dont on peut faire une analyse inspirée de la méthode critique historique pour discerner les hiérarchies implicites entre enseignants et historiens notamment, et les différences de légitimité à dire ce qu'il en est de cet enseignement.

Commençons par une critique externe. «La parole aux enseignants » : ainsi s'intitule la section du sommaire du numéro 175 prise en charge par des enseignants, encadrée par deux sections ( « Enseigner l'histoire de France » et « Chez nos voisins européens ») proposant des textes d'historiens (et d'inspecteurs généraux). La « parole » qu'on accorde aux enseignants relève du témoignage et se marque, par contraste avec les autres textes, par l'absence de notes de bas de page (et donc de références), quand les spécialistes proposent des analyses (historiques ou d'experts), ce qui manifeste une catégorisation - forte dans le champ historique - du témoin, de l'acteur et de l'historien : là où l'expert joue de son statut et le scientifique de ses références, l'enseignant-témoin n'a pour seule arme que son expérience individuelle ( Il me semble, d'après nos observations, que les écarts de maîtrise (notionnelle langagière, culturelle, relationnelle) chez les élèves se creusent de plus en plus », écrit ainsi un enseignant ayant 9 ans d'ancienneté, s'appuyant sur un collectif indéterminé - « nos observations », p. 133). Dans le numéro 177 , une rubrique intitulée « Le passé tel qu'on l'enseigne » est constituée de trois textes d'auteurs qui n'ont avec l'enseignement de l'histoire qu'un lien d'expérience et/ou d'intérêt « intellectuel »: Mara Goyet, enseignante d'histoiregéographie et essayiste; Bérénice Levet, philosophe; Vincent Badré, professeur d'histoire-géographie et essayiste. Ces auteurs n'appartiennent pas à une communauté de recherche sur les objets dont ils traitent, et ne fondent pas leurs analyses sur des études empiriques - malgré ce que laisse à entendre le titre de la rubrique ( le passé tel qu'on l'enseigne ») qui ne différencie pas l'expérience de l'étude empirique. Ce biais est censé être partiellement compensé, selon un procédé qui a cours dans le monde médiatique, par la consultation non contrôlée de ce qui est aisément accessible : les manuels. Prendre un support de l'activité pour en tirer des conclusions sur l'effectivité des pratiques constitue un raccourci classique dans le traitement des questions d'enseignement qui ne s'embarrasse pas de justification (Bruter, 2007). Ainsi commence l'article de Vincent Badré : « Les livres de classe ne sont pas un reflet exact de l'enseignement historique effectivement dispensé dans les collèges et les lycées. Ils sont cependant un assez bon reflet de ses tendances générales $»$. C'est précisément ce genre de hiatus que la démarche didactique vise à prendre en charge. La section « Manuels : impressions de lecture » du même numéro 177 annonce quant à elle, dès le titre, la nature des analyses proposées.

La lecture de ces témoignages offre, à l'échelle de leur contenu également (critique interne), une image des catégories de l'inconscient scolaire spécifique à 
l'enseignement de l'histoire. À propos de la formation qu'il a reçue, un enseignant rend compte à deux niveaux de la prégnance de la dichotomie des contenus et de la pédagogie. D'une part, l'analyse qu'il propose de son expérience juxtapose les contenus et le pédagogique dans des catégories très étanches: « Nous aurions souhaité nous initier aux techniques de communication publique, aux ressorts précis d'une pédagogie orale [...]. Enseigner clairement, captiver son auditoire, amener l'élève à la double injonction, difficile à faire tenir ensemble, de devenir critique et citoyen [...] ce tout forme une alchimie rare, enthousiasmante et digne d'effort $»\left(n^{\circ} 175\right.$, p. 134). Les catégories à l'œuvre ignorent toute condition sociale d'accès à la critique, et simultanément toute condition épistémologique aux interactions du maître et des élèves. D'autre part, de manière plus implicite, la relation de cet enseignant à la formation qu'il a reçue (au milieu des années 2000), renvoie davantage à une relation scolaire de type transmission / assimilation qu'à une formation universitaire. Relation qui maintient vivante la dépendance décrite comme subie de l'étudiant envers ses formateurs, qu'on voit poindre derrière certains reproches : « nous n'avons jamais eu la moindre enquête PISA en main [...], aucun débat sur ces sujets » (p. 134).

Poussée à son extrême, la dichotomie qui favorise les contenus au détriment de la pédagogie conduirait à ne plus distinguer l'enseignement scolaire de l'enseignement universitaire. La logique apparente de cette perspective mérite d'être rapportée aux conditions sociales de possibilité de la production scientifique. La circularité pointée par les travaux de Kuhn (1990) entre paradigme et communauté scientifique $^{21}$, entre dimension épistémique et dimension institutionnelle du travail des savoirs, pose à l'enseignement la question essentielle du changement de contexte : comment ne pas réduire le savoir historique à du savoir mort lorsqu'on l'extrait de sa communauté de production en en faisant un objet d'enseignement scolaire porté par des professeurs non historiens? Ainsi, l'énoncé qui a le statut d'hypothèse dans la communauté scientifique prend le statut de vérité lorsqu'il la quitte pour la classe. Quand bien même l'enseignant scolaire serait également chercheur en histoire, cette différence de statut qu'il percevrait serait difficile à dépasser avec des élèves. Et dans la plupart des cas, les enseignants scolaires sont soumis à des contraintes, notamment institutionnelles, qui les empêchent, la plupart du temps, de participer à une telle communauté scientifique ${ }^{22}$. Un moyen de considérer cette impasse apparente consiste à se pencher sur le sens de la dichotomie du contenu et de la pédagogie pour les enseignantschercheurs qui eux remplissent la double condition : ils enseignent et appartiennent à une communauté scientifique. L'étude menée par Pace (2004), historien américain au fait des travaux en history education, montre que la même dichotomie opère, sous une forme spécifique, chez les historiens dès lors qu'il s'agit de traiter de leur activité d'enseignement. Les historiens interrogés déclarent que leur liberté de recherche garantit la qualité et la pertinence de leur enseignement, mais également que l'activité de chercheur en histoire repose sur des procédures et des institutions bien stabilisées, alors que l'enseignement est une pratique qu'il serait difficile d'appréhender de manière systématique et donc scientifique, tant elle repose sur des individualités et des facteurs multiples (Pace, 2004, p. 1188). La dichotomie est en l'espèce référée à une différence de nature épistémologique qui séparerait deux types de recherche : sur le passé et sur les pratiques d'enseignement, sans que cette séparation ne soit justifiée (qu'est-ce qui permettrait d'expliquer la possibilité d'étudier scientifiquement les pratiques des hommes du passé, mais pas les pratiques d'enseignement?). La dichotomie est donc ancrée de la même manière chez les enseignants et chez les enseignants-chercheurs, même si leurs discours de justification sont différents.

\section{Sciences de l'éducation et didactique dominées}

Le peu de cas fait des activités d'enseignement comme objet d'étude potentiel renvoie à une hiérarchie implicite des disciplines universitaires qui positionne les sciences de l'éducation, et parmi elles les didactiques disciplinaires, vers le bas de l'échelle. Cette hiérarchie - avérée par des études de longues séries de données récentes pour le cas français notamment (Renisio, 201523) - pour être appréhendée en tant que structure cognitive dans l'inconscient des agents, nécessite d'être observée à travers des situations empiriques dans lesquelles elle manifeste ses effets.

\section{Les sciences de l'éducation doublement dominées}

Si les travaux de recherche qui prennent pour objet les sciences de l'éducation sont peu nombreux, 
l'étude déjà ancienne proposée par Fournier, Gingras et Mathurin (1988) donne cependant à voir comment la dichotomie contenus / pédagogie peut se manifester dans de multiples directions qui entrent parfois en contradiction. Le cas du processus d'évaluation (en vue d'une promotion) d'un professeur d'une faculté de sciences de l'éducation du Québec met en lumière les tensions liées à la définition du poids de la recherche et du type de recherche légitime, lorsqu'il s'agit d'évaluer une double activité d'enseignement et de recherche. Quand l'instance d'examen du dossier est interne à la faculté des sciences de l'éducation, le jugement par les pairs est défavorable à la promotion et s'appuie sur une définition non disciplinaire et indépendante des contenus de la recherche : ce professeur ne peut espérer devenir enseignant-chercheur à part entière car ses recherches ne satisfont pas aux critères d'une norme scientifique non écrite, mais indexée aux disciplines les plus légitimes de l'Université. En revanche, lorsque l'instance d'examen est constituée de professeurs de différentes facultés, elle retourne à la légalité, aux textes qui régissent ces promotions et qui définissent la spécificité de la recherche dans le domaine des sciences de l'éducation à laquelle se conforme le candidat. Les auteurs de l'étude suggèrent que ces deux perspectives s'expliquent notamment par la position des sciences de l'éducation dans le champ universitaire : « la position de la Faculté des sciences de l'éducation est paradoxale : elle est la moins professionnelle des facultés dites professionnelles (médecine ou droit) et la moins scientifique des facultés ou départements scientifiques. Cette situation particulière oblige les professeurs à répondre simultanément et/ ou successivement à des exigences contradictoires » (Fournier et al., 1988, p. 48). D'où des prises de position parfois radicales des enseignants-chercheurs de cette discipline afin de renforcer une légitimité que l'ethos de la communauté scientifique place au niveau de la recherche plutôt que de l'enseignement. Le point saillant dans l'étude de cette situation réside dans la facilité avec laquelle la catégorie disciplinaire (sciences de l'éducation) est abandonnée lorsque la construction de la légitimité au sein de l'université est en jeu. Abandon diamétralement opposé au poids habituellement donné à l'organisation disciplinaire lorsqu'il s'agit de penser l'enseignement scolaire et la formation des enseignants. Par un renversement radical, la pratique de la recherche dans ses dimensions théoriques devient une norme absolue qui transcende l'organisation disciplinaire : il est reproché à ce professeur une activité de recherche « liée à des projets d'intervention », une " recherche à portée pratique », sur les pratiques pédagogiques, et non sur les théories de son champ disciplinaires (Fournier et al., 1988, p. 49). La dichotomie contenus / pédagogie agit alors à deux niveaux : elle se confond avec la dichotomie théorie / pratique au sein de l'activité de recherche, et en faveur de la théorie, et elle minore l'activité d'enseignement. Les auteurs de l'article montrent à travers la question évaluative $\mathrm{du}$ « rayonnement » du professeur que l'enjeu de la définition de la recherche porte sur le périmètre de la communauté concernée : soit réduite aux seuls chercheurs, soit ouverte au-delà vers les praticiens; autrement dit, il porte sur le poids respectif de l'évaluation par les pairs et de l'utilité sociale de la recherche.

\section{La recherche didactique ignorée}

Les recherches en didactique de l'histoire, à l'instar de ce cas en sciences de l'éducation, pour récentes qu'elles soient, font l'objet d'une indifférence, voire d'un rejet, tant de la part des enseignants que d'autres acteurs, universitaires ou non, qui prétendent dire ce qu'il en est de l'enseignement et de l'apprentissage ${ }^{24}$.

Les deux numéros de la revue Le Débat de l'année 2013 précédemment évoqués témoignent en creux du rejet. L'enseignement et l'apprentissage, objets de discussion, n'y sont jamais des objets d'étude, et sont confiés soit à des témoins d'expérience (les enseignants), soit à des chercheurs de disciplines hiérarchiquement mieux placées (l'histoire en premier lieu, mais aussi la philosophie et la sociologie). Cependant, on l'a évoqué, la domination des historiens repose aussi sur leur expérience d'enseignants, et pas seulement sur leur appartenance à une discipline universitaire ancienne et légitime. Cette double légitimité conduit à éluder la question de leur légitimité à traiter d'enseignement, dans un processus qu'on pourrait rapprocher du rejet par une partie des chercheurs des études de sociologie de la science ${ }^{25}$. Ce traitement, illustré ici par une revue "d'analyse et de discussion », comme se définit Le Débat, correspond à la manière dominante dont la perspective didactique en histoire est considérée, ou plutôt ignorée, dans les débats publics. Comme pour le sociologue de la science, le problème posé par le didacticien consiste à constituer en objet la pratique de ceux qui habituelle- 
ment objectivent les autres : les historiens objectivent les pratiques des hommes du passé, les professeurs objectivent les pratiques des élèves. Cette incongruité est renforcée par la proximité des observateurs (didacticiens) et des observés (enseignants) : ils sont finalement très semblables alors que la distance est évidente entre l'historien d'aujourd'hui et l'homme du passé, entre le professeur (adulte et instruit), et l'élève (enfant et en cours d'instruction). Proximité d'autant plus forte pour la didactique de l'histoire que la très grande majorité des didacticiens sont d'anciens enseignants scolaires, et enseignent eux-mêmes (dans leurs institutions de formation).

\section{Une ouverture par l'historiographie et l'épistémologie}

On peut cependant trouver des exceptions à cette posture dominée des recherches en didactique de l'histoire. Un dossier publié par la revue Les Annales en 2015, et un article collectif de la revue d'histoire Vingtième Siècle reprennent, à destination d'un public d'historiens et de chercheurs en sciences sociales, les enjeux de formation et de recherche : la professionnalisation des enseignants, et l'opposition entre recherche appliquée et recherche fondamentale. Les analyses proposées dans ces articles ne constituent pas des études empiriques sur l'enseignement ou la formation des enseignants d'histoire, mais cependant des approches scientifiquement situées : si on y trouve, comme dans Le Débat, des témoignages, les analyses principales relèvent des sciences sociales et historiques et de l'épistémologie (De Cock, 2015; Delacroix, 2015; Girault, 2015; Bourdon et al., 2015). D'une part, ces regards peuvent permettre de dépasser la dichotomie des savoirs et des pratiques pour en appréhender les relations, et d'autre part, ils autorisent un croisement disciplinaire avec la didactique - sorte d'épistémologie appliquée - que l'on constate par les références faites à des concepts et études didactiques ${ }^{26}$, qui ouvrent un dialogue possible, notamment par le biais de l'historiographie et de l'épistémologie de l'histoire comme champs d'intersection, pour penser la formation des enseignants d'histoire. Ainsi, Delacroix incite-t-il à mettre en question la faible légitimité des savoirs didactiques:

«Encore faudrait-il interroger la construction de cette opposition et donc son histoire pour essayer de comprendre la persistance chez de très nombreux collègues universitaires historiens d'une sorte de méfiance voire, pour certains, d'aversion envers la "pédagogie" quand elle entre dans le périmètre universitaire, y compris dans le cadre des départements des sciences de l'éducation. Les travaux qui s'y rapportent (qu'ils soient qualifiés de didactiques ou non) sont rejetés hors de la sphère de la légitimité scientifique disciplinaire et cantonnés dans celle des savoirs pratiques (voire quasi techniques) de transmission. Cette séparation est pensée sur le modèle de celle qui est établie entre le scientifique (noble et désintéressé) et le technique (utilitaire). Ce dualisme est donc de nature épistémologique, une dimension qui reste finalement assez peu prise en compte dans le débat entre "partisans des savoirs" et pédagogues. » (Delacroix, 2015, p. 200-201).

Il est en outre notable que dans son étude historique des concours de recrutement, Delacroix (2015) mette d'abord en lumière l'opposition entre le scientifique et le pédagogique, avant d'introduire (p. 193) un troisième terme, à propos de la réforme de 1993 : c'est alors moins le "disciplinaire », le "scientifique » ou encore les « contenus » que l'épistémologie et l'historiographie qui s'opposeraient au pédagogique et professionnel. Ce faisant, il parle d'un « tournant épistémologique 27 » (p. 194), par opposition avec le titre de l'article - « tournant pédagogique »-qui marquerait la réforme récente, et oppose de manière parfois polémique le professionnel (pédagogique et civique) à la dimension réflexive que porteraient épistémologie et historiographie; dimension charnière dans le texte de B. Girault également. C'est sans doute sur cette " réflexivité » que didactique, d'une part, épistémologie et historiographie d'autre part, peuvent entrer en dialogue, ne serait-ce qu'en poursuivant le processus de différenciation mené par Delacroix, mais du côté de la dimension «professionnelle » de la formation cette fois-ci, en distinguant pédagogique et didactique ${ }^{28}$.

\section{Lobstacle des représentations didactiques des enseignants}

Si l'on peut être d'accord avec Bourdon et al. et Delacroix sur le fait qu'épistémologie et historiographie sont indispensables pour permettre à chaque professeur de comprendre de manière critique les choix qui ont été faits dans les programmes, il nous 
semble qu'elles peuvent, bien au-delà, jouer un rôle de dépassement de la dichotomie des savoirs et de la pédagogie, à condition de penser la formation des enseignants au-delà du seul concours de recrutement ${ }^{29}$. Elles peuvent en effet fournir les connaissances et les repères pour mettre au travail l'analogie $\mathrm{du}$ rapport entre savoirs et pratiques de mise en texte des savoirs, chez les historiens et en classe. Par exemple, lorsque des textes d'aide à la mise en ouvre des programmes comme dans la fiche " ressource » déjà évoquée pour la classe de $2^{\text {nde }}$ qui fait allusion à la micro-histoire et à l'étude des civilisations en termes braudéliens pour discuter les enjeux de la généralisation dans les "études ciblées » destinées aux élèves, les professeurs doivent être armés pour en faire une analyse critique. Autrement dit, ils doivent pouvoir resituer les concepts de représentativité et de généralisation qui sous-tendent ces études ciblées dans les débats (cristallisés notamment à la fin des années quatre-vingt) et aux ouvertures auxquelles ces derniers ont mené (la micro-histoire, et même au-delà selon Chartier, 1989 et Revel, 1996), dans leur continuité avec les mouvements historiographiques, de l'enchaînement linéaire de l'analyse critique et de la synthèse de l'école méthodique (Langlois et Seignobos, 1898) à l'éclipse de la synthèse au profit d'une histoire-problème qui prend ensemble analyse et explication ${ }^{30}$.

Ces perspectives posent le problème de la formation des enseignants d'histoire en incitant à l'envisager au-delà de la déploration d'un manque de connaissances épistémologiques et historiographiques que démentent d'ailleurs des constats comme ceux de B. Girault (2015, p. 210) de la difficulté des enseignants en formation de mettre à profit leurs connaissances avérées dans les domaines historiques auxquelles les préparent le concours du CAPES. Constats qui soulignent que l'enjeu de formation réside plutôt dans les conditions d'usage de ces savoirs épistémologiques et historiographiques dans les situations d'enseignement. On peut faire l'hypothèse qu'il s'agit moins d'un problème d'ignorance que de représentations didactiques ancrées dans la dichotomie savoirs / pratiques, qui constituent de véritables obstacles épistémologiques (Bachelard, 1938) pour ces futurs enseignants, obstacles dont la caractéristique principale est d'être résistants même aux meilleurs raisonnements logiques. Le retour à l'étude de la situation de formation menée par Seixas (1999) fournit des pistes pour penser ce rapport entre représentations didactiques des enseignants et formation.

\section{UNE SITUATION DE FORMATION QUI FAIT CAS AVEC L'INCONSCIENT SCOLAIRE}

\section{Former aux résultats ou former à l'enquête scientifique}

À l'occasion d'une session de formation continue d'enseignants organisée autour d'interventions de chercheurs en sciences sociales et en histoire aux États-Unis, Seixas (1999) observe et mène des entretiens avec les différents protagonistes dans une perspective didactique. Un historien déclare ainsi faire une « conférence sur les contenus», qu'il justifie par le besoin qu'il suppose chez les enseignants de savoir « ce qui se passe dans le champ de l'histoire». Il explique que dans ce but, il adapte son discours à ce public particulier, conjecturant que les enseignants « sont probablement peu habitués au genre de débats que nous avons dans les cercles professionnels, qui sont une destruction complète des récits, et de toute construction de la réalité et de la vérité. [...] Je veux dire que quand j'ai mon autre casquette, je peux me réjouir du fait que l'histoire est dangereuse, que l'histoire est problématique, que c'est complètement déstabilisant pour de très bonnes raisons. Mais en venant ici, soulever ces questions ou ouvrir la boîte de Pandore, je ne suis pas certain que c'est ce que je suis supposé faire ici » (Seixas, 1999, p. 322). D'où son interprétation restrictive de "ce qui se passe dans le champ de l'histoire », qui se résume à transformer des hypothèses et des pratiques de recherche en contenus.

\section{L'enquête enseigne les savoirs}

Les choix ainsi justifiés par cet historien prennent sens dans la situation de son intervention, c'est-àdire en fonction du public d'enseignants auquel il s'adresse en tant que chercheur et à la représentation qu'il a de leur rapport au savoir et à la recherche historiques. Or le point de vue des enseignants auditeurs observés par Seixas révèle une insatisfaction d'en rester à une litanie de contenus. Insatisfaction qui apparaît en particulier à l'occasion d'une conférence qui sort du format dominant illustré par le discours 
de l'historien, et qui questionne ce que « contenus» signifie dans ce type de situation : cette intervention fait cas pour les enseignants, et constitue en ce sens une situation didactiquement problématique. Cette conférence d'une anthropologue, à propos d'un parc d'attraction aquatique envisagé comme construction culturelle, n'a pas apporté de contenus dans le sens précédent du terme, mais « c'était plutôt sa méthode, ses procédés, sa stratégie qui ont constitué le contenu utile» (Seixas, 1999, p. 325) pour les enseignants auditeurs. L'intéressant selon eux réside non dans un contenu disciplinaire ou pédagogique, mais dans l'exposé de l'enquête en train de se faire et de ses détours, des moyens mis en œuvre pour saisir le parc d'attraction comme une source de l'enquête sur la société américaine d'aujourd'hui. Pour Seixas, l'intérêt porté par les enseignants à cette conférence s'explique par le fait « que le contenu de la conférence de Davis [l'anthropologue] était de la pédagogie. Par le biais de sa recherche, elle enseignait à l'auditoire comment apprendre en examinant une source, et en montrant le type de questions qui peut émerger de cette source singulière » (Seixas, 1999, p. 327). Le constat du pédagogique ainsi intégré (embedded) au contenu nous paraît intrinsèquement lié à la situation de formation qui met aux prises des enseignants scolaires et une chercheuse dont l'expérience de recherche constitue pour eux un « exercice exemplaire ${ }^{31} »$ d'enquête, telle qu'ils pourraient l'envisager pour leurs élèves : non pas une théorisation du travail d'enquête mais une description des moments d'incertitude et des choix relatifs auxquels s'est trouvée confrontée l'anthropologue. Se cristallise alors dans la situation de formation le rapport entre la recherche en anthropologie comme source de formation des enseignants, et les conditions d'apprentissage par les élèves du travail d'enquête anthropologique. On lit dans les termes de Seixas la continuité entre recherche et enseignement : l'explication de l'enquête constitue une occasion de penser comment apprendre de l'enquête anthropologique, autrement dit comment les chercheurs s'enseignent les uns les autres ${ }^{32}$.

On retrouve dans cette continuité la perspective profondément pédagogique de l'épistémologie de Bachelard, qui rend compte des enjeux de l'apprentissage scientifique en terme de dialectique entre l'intériorisation des normes et l'extériorisation dans la communauté scientifique ${ }^{33}$. Sa psychanalyse de la connaissance met au jour le rôle central de la « surveillance intellectuelle de soi » (Bachelard, 1949) qui s'incarne dans le dialogisme de l'activité scientifique : le savant écrit toujours avec un lecteur de sa communauté au-dessus de l'épaule, ce qui en fait un individu tout à la fois maître et élève. Dans ce processus, c'est l'existence effective et la conscience de l'existence de la communauté objectivée dans ses outils et ses publications qui donnent sens à ce dialogisme ${ }^{34}$. "Faire école en soi » n'est cependant possible qu'en rapport avec une extériorisation formatrice de cette surveillance. C'est la raison pour laquelle il est nécessaire, pour Bachelard, de prendre au sérieux l'adage qui dit qu'enseigner est la meilleure façon d'apprendre (Bachelard, 1949, chap. II), ce qui renverse les conditions habituelles des relations du maître à ses élèves. En montrant l'anthropologue comme maître qui se fait l'élève expliquant sa démarche d'enquête, et les enseignants comme élèves qui se font maîtres en évaluant cette explication du point de vue de leur enseignement, la situation étudiée par Seixas induit deux conséquences.

\section{Une étude de cas qui ouvre le champ des possibles}

D'une part, elle illustre la manière dont on peut faire émerger d'une situation de formation des éléments d'interprétation des catégories naturalisées des agents, à condition de rapporter des cas comme celui-ci, aux interactions habituelles des enseignants et des chercheurs. Dans un tel processus, le didacticien ne se limite pas à produire des règles qui décrivent le fonctionnement ordinaire des interactions entre enseignants en formation et chercheurs, il part des possibles habituellement mis en œuvre, pour reconstruire le champ des possibles des uns et des autres étoffé de « ce qu'ils pourraient faire mais qu'ils ne font pas ou du moins pas ordinairement » (Bazin, 2008, p. 356). En s'appuyant ainsi sur ce qui fait cas, le statut des règles comme régularités peut se modifier pour éviter à l'observateur de « glisser du modèle de la réalité à la réalité du modèle » (Bourdieu, 2000a, p. 253) : les sorties des régularités manifestent que ces règles ne s'appliquent pas mécaniquement mais sont problématiques dans leur rapport aux situations parce qu'elles portent les stratégies des agents. Non seulement une telle situation constitue un microcosme social et institutionnel qui met en suspens les lois ordinaires des relations entre 
enseignants scolaires et chercheurs, mais elle permet au travail didactique de donner à voir les conditions sociales de l'émancipation de ce rapport ordinaire de domination.

\section{L'hypothèse d'une formation par exemples exemplaires de construction et résolution de problèmes didactiques}

D'autre part, cette étude suggère le rôle que peuvent jouer des « exercices exemplaires» dans la formation à l'enseignement, et qui apparaissent comme des liens potentiels entre le singulier de la situation de formation et le développement du métier d'enseignant. On l'a vu, le récit d'une enquête rapportée par l'anthropologue aux enseignants, avec ses incertitudes et ses problèmes, constitue le support de la formation dans sa double dimension de contenu et de pédagogie. Surtout, l'idée d'exercices exemplaires fait écho aux paradigmes selon la définition finalement privilégiée par Kuhn d'exemples exemplaires $^{35}$ (exemplars), qui renvoie explicitement dans ses textes à la formation à la recherche des « jeunes» chercheurs (Kuhn, 1983, p. 255-260). Ce concept, dont la potentialité didactique a été soulignée par Sensevy ${ }^{36}$, met en lumière une approche de la formation masquée par la dualité de la recherche et de l'enseignement, y compris dans des études comme celle de Pace (2004) sur les enseignants-chercheurs en histoire : ces derniers peuvent pratiquer l'enseignement par le biais de deux modalités radicalement différentes selon qu'il s'agit de l'enseignement dans les premiers cycles universitaires ou de la formation des doctorants (notamment par le biais de séminaires). Dans la situation de formation étudiée par Seixas, ce qui fait cas c'est que l'intervention de l'anthropologue rend possible la deuxième forme, alors même que la situation institutionnelle correspond à la première : en faisant le récit problématique de son enquête, l'anthropologue ne théorise pas le travail anthropologique selon des règles de méthode, mais elle donne à voir une situation d'enquête effective dont il s'agit pour les enseignants de travailler les ressemblances avec les situations de classe qu'ils pourraient proposer à leurs élèves. Il ne s'agit alors plus de voir la formation en question comme la tâche, incombant au seul formé, de faire entrer ses situations de classe dans les règles d'une méthode générale, mais bien plutôt d'apprendre par ce cas d'enquête problématique exposé, à voir les ressemblances $^{37}$ avec ses propres problèmes didactiques. En l'espèce, l'expérience d'enquête anthropologique relatée est exemplaire par son intégration des contenus et de la pédagogie qui rend possible un travail des ressemblances avec les dispositifs d'enseignement mis en ouvre dans leurs classes par ces enseignants, parce qu'elle est exemplaire du travail d'enquête en sciences sociales. Mais elle est simultanément exceptionnelle par rapport aux pratiques didactiques habituelles de ces enseignants. Pivot de la dialectique de la tradition et de l'innovation (Kuhn, 1990, chap. IX), le paradigme comme exemplar peut jouer le rôle d'outil conceptuel essentiel pour penser les relations dynamiques entre structures cognitives et structures institutionnelles dans l'enseignement de l'histoire et dans la formation des enseignants.

Cette idée d'exercices exemplaires, mise en perspective par les travaux de Kuhn, nous semble être la piste la plus intéressante qu'offre la proposition de Seixas. Cependant, la situation étudiée reste ponctuelle, et par là s'écarte des enjeux problématiques du concept de Kuhn qui associe les exemples exemplaires à une communauté. Bien que ce type d'expérience de formation soit en mesure de marquer les participants par son exceptionnalité, elle ne saurait se substituer au long travail d'acculturation que suppose l'apprentissage par exemples exemplaires qui correspond aussi à l'intégration à une communauté scientifique constituée. C'est sans doute la raison pour laquelle Seixas semble céder à la facilité du courtcircuit pour imaginer les effets durables de l'expérience : à propos de ce que dit un des organisateurs de la formation des limites des interventions des historiens et de l'anthropologue lors d'un entretien, Seixas estime qu'il « regardait dans la mauvaise direction : la pédagogie qu'il cherchait était intégrée dans le contenu apporté par Davis. Ce qu'il fallait c'était quelqu'un qui aurait articulé la méthode de Davis et montré comment le même genre de critique pourrait être entreprise avec des élèves » (Seixas, 1999, p. 327) ; on imagine que ce quelqu'un n'est autre que le didacticien lui-même, pensé ici comme en mesure, par son discours, de montrer la voie. L'idée d'un intercesseur capable de transformer l'occasion en apprentissage nous paraît oublieuse de la vigueur des représentations didactiques des enseignants, de leur caractère d'obstacle épistémologique, et des conditions de possibilité liées au concept d'exemple exemplaire. 
Des exemples exemplaires didactiques pour mettre au travail les obstacles épistémologiques des enseignants

On gagne en effet à envisager les conséquences de cet ancrage des représentations des enseignants dans les termes de la rupture épistémologique qui va avec l'idée d'obstacle chez Bachelard, en observant avec Orange (2006) qu'enseignants et chercheurs en didactique sont soumis à des « cadres d'analyse mobilisés implicitement ou explicitement » (p. 122) qui guident l'interprétation des situations d'enseignement-apprentissage. Si l'intérêt des enseignants et celui des chercheurs en didactique convergent en direction des analyses qui portent sur les relations entre les pratiques de classe et les apprentissages des élèves, les cadres dans lesquels s'effectuent ces analyses divergent : dans une perspective bachelardienne, il existe une véritable rupture didactique entre les deux (Orange, 2006); de quel ordre est cette rupture?

Elle peut d'abord être appréhendée par l'idée d'obstacle épistémologique. Les cadres mobilisés par les enseignants pour effectuer leurs analyses, nécessaires à leur activité quotidienne, ne peuvent être aisément mis en question du fait de leur efficacité : ils sont éprouvés par l'expérience. Des solutions différentes peuvent être comparées et discutées, mais elles le seront dans un cadre qui reste stable pour chacun des interlocuteurs. Or une des caractéristiques de l'activité scientifique est précisément de s'inscrire dans des cadres d'analyses spécifiques, propres à chaque communauté. Par là, les enseignants qui eux ne s'inscrivent pas dans une communauté scientifique sont dépendants de cadres d'abord liés à leur expérience. À ce niveau, la rupture est donc liée à la pratique ou l'absence de pratique scientifique qui différencie la nature même des cadres d'analyse en jeu.

La rupture renvoie, à un second niveau, à la relation au cœur de la problématique de Kuhn, entre la dimension épistémologique du cadre et sa dimension institutionnelle (la communauté). On peut donc envisager de s'appuyer sur les concepts de $\mathrm{Kuhn}^{38}$ pour explorer ces questions didactiques, et en particulier les composantes des matrices disciplinaires : généralisations, modèles ou croyances, valeurs, et exemples exemplaires (Kuhn, 1983, p. 248-255). Tout d'abord, le rôle central qu'il accorde à ces derniers permet de formuler une hypothèse de formation par « exemple exemplaire »: la rupture entre un cadre d'analyse d'enseignant et un cadre d'analyse de didacticien, c'est-à-dire la possibilité de mettre en question les représentations didactiques, passe par un changement de communauté (par intégration à une autre communauté ou transformation d'une communauté existante) entendu comme le développement de la maîtrise d'exemples exemplaires de construction et résolution de problèmes didactiques.

En second lieu, la différenciation entre généralisations, croyances, valeurs et exemples exemplaires permet de mieux caractériser les cadres d'analyse des enseignants et des didacticiens, et de les différencier. Non seulement le cadre détermine l'analyse des relations entre pratiques d'enseignement et apprentissages des élèves, mais il détermine simultanément les interprétations des données disponibles sur ces apprentissages, qui ne sont pas accessibles directement (ils reposent sur des productions d'élèves qu'il faut interpréter si l'on veut y appréhender des activités intellectuelles, Orange, 2006, p. 123). La différenciation des composantes d'une matrice disciplinaire nous outille pour développer ces deux plans de détermination du cadre d'analyse didactique. D'une part, il détermine l'analyse des relations entre pratiques et apprentissages par le biais de " généralisations » sous forme de "lois » ou de "règles » (Kuhn, 1983, p. 248-249), telles que, par exemple, la " généralisation » constructiviste qui définirait la règle suivante : une activité dans laquelle l'élève est amené à construire une réponse permet un apprentissage. D'autre part, le cadre d'analyse détermine la relation entre ce type de généralisations (à propos de la relation pratiques / apprentissages), et la production des données (sur l'apprentissage notamment, mais aussi sur les pratiques) qui les rend opératoires. Mais cette seconde opération est de nature différente de la première parce qu'elle met en jeu des manières habituelles et légitimes de produire des données, qui renvoient aux exemples exemplaires de résolution d'énigme de la communauté scientifique concernée. Se manifeste alors la différence de nature entre les cadres du professeur et du didacticien : là où le professeur, en l'absence de manière exemplaire de faire, sélectionne des données parmi les productions des élèves en fonction des idées explicatives (généralisations, croyances ou valeurs) qu'il a sur la relation pratiques / apprentissage, le didacticien, parce qu'il en a le loisir ${ }^{39}$ et l'expérience au sein de sa communauté de recherche, produit des données en fonction 
des « ressemblances» qu'il identifie, dans la situation, avec d'autres situations d'analyse dans lesquelles il a pu mettre en œuvre des manières "autorisées » (Kuhn, 1983, p. 258) par sa communauté parce qu'exemplaires. Cette opération - production vs sélection - se distingue de celle du professeur parce qu'elle engage la relation entre données empiriques et idées générales explicatives au sein de manières habituelles de faire qui l'encadrent et permettent leur mise en tension, alors qu'en l'absence d'exemples exemplaires de manières de faire, les idées explicatives neutralisent la production critique des données.

De là une reformulation possible de l'hypothèse « exemple exemplaire ». Les conditions d'une rupture avec les représentations didactiques se situent au niveau de l'insertion dans une communauté définie par le rôle central qu'y jouent les exemples exemplaires de production et de résolution de problèmes didactiques; ces exemples exemplaires mettent en jeu des opérations scientifiques qui ne se limitent pas à la confrontation de théories (sous la forme de « généralisations » notamment) ni à la sélection de données appropriées à une argumentation, mais engagent potentiellement une production réciproque, et par là critique, de données et d'idées explicatives. Nous proposons de faire une exploration de cette hypothèse à travers une dernière étude de cas.

\section{UNE OCCASION : L'INTRODUCTION DE LA RECHERCHE DANS LA FORMATION}

L'introduction de la recherche dans la formation des enseignants français lors de sa transformation récente (dite de "masterisation ${ }^{40}$ ", 2010) constitue une occasion pour explorer cette hypothèse. Elle met en effet les structures cognitives des élèves, des professeurs et des didacticiens aux prises avec les structures institutionnelles de la discipline scientifique, de sa didactique et de son enseignement en classe. De ce fait, l'émergence de la recherche dans la formation rend effective sur le plan institutionnel la question de la relation des enseignants à une communauté de recherche et à ses exemples exemplaires de construction et de résolution de problèmes, et modifie ainsi potentiellement le rapport des apprentis enseignants à la formation et aux formateurs. Elle interpelle en outre ces derniers dans leur propre rapport à la recherche didactique et à la recherche disciplinaire en reposant la question de l'étude de Fournier et al.
(1988) sur la définition et le rôle de la recherche dans une composante universitaire de formation professionnelle. En l'espèce, la recherche dans un master professionnel et disciplinaire ${ }^{41}$ est en décalage avec le rôle qu'elle joue dans un master dont la visée principale est de préparer à la pratique de recherche dans une discipline : bien qu'elle soit disciplinaire (didactique de l'histoire), elle porte sur l'activité professionnelle (enseigner) et ne vise pas à faire de la recherche l'activité professionnelle (ni en histoire, ni en didactique), ce qui rend problématique son existence pour les étudiants concernés ${ }^{42}$, et sa justification pour les formateurs $^{43}$. Mais ce questionnement est analogue à celui de l'enseignement scolaire de l'histoire qui ne vise pas à faire des élèves de futurs historiens, mais de futurs citoyens. L'écart entre apprendre l'histoire pour devenir historien et apprendre l'histoire pour devenir citoyen constitue bien souvent un implicite, comme si le pendant scolaire au métier d'historien était pour les élèves le «métier » d'historien scolaire (qui consisterait notamment à produire des textes à partir de documents, selon le format structurant des examens, brevet et baccalauréat). À ces deux niveaux, l'ambiguité est forte et rarement posée comme telle : quel est l'intérêt d'initier (des enseignants, des élèves) à une pratique de recherche scientifique? Si les futurs enseignants ne sont pas destinés à devenir chercheurs en didactique mais praticiens de la didactique, comment justifier qu'on les initie à la recherche en didactique?

\section{Un cas d'initiation à la recherche pour penser l'hypothèse " exemple exemplaire "}

Le cas d'une étudiante intégrée à un séminaire de recherche dans le cadre de son master (deux années) pour devenir enseignante d'histoire-géographie dans le secondaire permet d'exploiter cette occasion que constitue la mise en place d'une nouvelle formation, à même de rendre visibles les tensions en jeu : d'abord étudiante, elle s'initie à la recherche et, en deuxième année, elle devient en plus praticienne (stage qui représente un tiers de son temps hebdomadaire), autant de rôles qui s'entrecroisent de manière parfois conflictuelle. Son travail de recherche s'effectue dans le cadre universitaire d'un séminaire piloté par un didacticien qui est le directeur de recherche ${ }^{44}$ des cinq étudiants qui y participent. Outre les cinq réunions annuelles du séminaire, le 
travail est matérialisé par des échanges de textes (et de commentaires sur les textes) avec le directeur.

Le choix de cette étudiante répond à la logique d'étude de cas qui se différencie de l'exemple ou de l'illustration par son rapport questionnant au « paradigme épistémologique dominant » : le cas « souligne les contradictions internes d'une norme ou les contradictions entre deux système normatifs [...] [il] pose une question sans pouvoir donner la réponse » (Ginzburg, 2010, p. 361-362). Le corpus correspondant au travail de cette étudiante fait cas parce qu'il donne accès aux effets de son inconscient scolaire disciplinaire, habituellement invisible parce que consubstantiel à son activité d'étudiante et d'enseignante: dans l'activité de recherche didactique qu'elle mène à terme - c'est-à-dire qu'elle déploie une problématisation didactique - elle donne à voir des éléments fondamentaux de cet inconscient scolaire. Elle met en discussion le paradigme épistémologique dominant de la dichotomie pratique / savoirs, sous l'aspect de l'apprentissage pensé comme imitation d'un modèle de (bonne) pratique enseignante; la production de son mémoire didactique la conduit à identifier l'obstacle que constitue ce modèle, car elle appréhende peu à peu que ni la tradition de la discipline scolaire, ni le modèle théorique proposé par transposition des pratiques historiennes n'est satisfaisant en tant que modèle de pratique d'enseignement. La solution qu'elle envisage, nous le verrons, est proprement didactique et marque la spécificité de ce domaine de recherche au sein des sciences sociales. Elle consiste à dépasser l'obstacle par l'abandon de la dichotomie en s'intéressant moins à l'exemplarité de la pratique qu'à celle de l'analyse de la pratique dans sa relation aux élèves et aux savoirs en jeux.

\section{En première année, une adhésion théorique à la référence historiographique}

Elle débute son travail de recherche en première année (M1) sur un plan essentiellement théorique et bibliographique, en cohérence avec la faiblesse de son expérience (la première année de master permet la mise en œuvre de quelques séances d'enseignement seulement, accompagnées par un professeur) et la place dominante des cours et travaux dirigés dans la formation. Son mémoire en fin de M1 (intitulé Didactique de l'étude de cas. Réflexions épistémologiques et application dans l'enseignement) constitue une réflexion épistémologique sur certaines perspectives historiographiques (le rôle de l'étude de cas dans la production de l'explication historique) et du questionnement de leur usage dans l'enseignement. Ce rapprochement entre épistémologie, historiographie et enseignement est une démarche organisée dans le séminaire comme entrée dans la recherche en didactique. La démarche est parallèle aux cours de didactique, qui visent l'appropriation d'une perspective didactique sur les phénomènes d'enseignement-apprentissage, et plus particulièrement du cadre théorique de l'apprentissage par problématisation (Fabre, 2009; Orange, 2012). Son sujet est ainsi délimité et développé à partir d'éléments travaillés en cours de didactique ${ }^{45}$, sur la base de la comparaison proposée entre étude de documents en classe et étude de cas chez les historiens (Ginzburg, 2010, p. 351-364), comme manière de penser la transposition didactique des pratiques historiennes de construction de problème.

Le parcours de cette recherche préliminaire s'engage de manière très classique en direction d'une thématique historique (la décolonisation marocaine) issue d'un travail de recherche antérieur en histoire (la décolonisation marocaine au regard de Ouest-France, 1953-1956), et adapté à une question d'enseignement (la place accordée à la décolonisation marocaine dans l'enseignement de la décolonisation dans le secondaire). Ainsi formule-t-elle son problème lors d'un séminaire du milieu de l'année de M1 : il s'agit de voir « comment faire comprendre la complexité aux élèves »; puis vers la fin de l'année : « comment rendre compte de la complexité avec l'étude de cas ». Initialement, son adhésion à la thèse de l'efficacité potentielle des études de cas est fondée sur des contraintes (de temps, de capacité et de connaissances des élèves) largement admises dans la communauté professionnelle des enseignants d'histoire du secondaire, qui se traduisent par la nécessité de travailler sur des études ciblées plutôt que de viser une impossible exhaustivité. Voici comment elle explique, en Ml, son ralliement à cette thèse :

«Un peu de recul suffit pour se rendre à l'évidence: un élève ne peut avoir connaissance de la totalité des faits historiques concernant la décolonisation, ce serait trop long, et ça n'aurait aucun intérêt. Le but de l'enseignement de l'histoire dans le secondaire est ailleurs. Une large communauté d'enseignants, de chercheurs, d'inspecteurs d'académie adhère à la 
multiplication des études ciblées dans l'enseignement de l'histoire pour permettre un approfondissement que ne permettrait pas un récit linéaire qui se voudrait le plus exhaustif possible. L'étude de cas peut alors être un procédé idéal pour que l'élève "s'approprie" un savoir en partant de la base, c'est-à-dire la source qui est au cœur du travail de l'historien, afin de "faire comprendre que l'histoire n'est pas écrite une fois pour toute mais qu'elle reste une construction", comme l'énonce le programme de la classe de Seconde. » (conclusion du mémoire de $\mathrm{Ml}$ )

Elle consolide en parallèle ses raisons d'adhérer à cette thèse sur un plan plus épistémologique dans les cours de didactique, en relation avec la situation ordinaire de la formation - et son rapport dominant / dominé - qui la rend peu légitime à discuter les thèses enseignées. L'approche par l'étude de cas est présentée et travaillées en cours comme une possibilité de pratique d'enseignement permettant la problématisation des élèves, fondée sur une transposition des pratiques savantes ${ }^{46}$.

Ses lectures et les échanges (de textes avec le directeur de recherche, et oralement lors des séminaires) aboutissent à une transformation des questions initiales en questions didactiques, qu'elle formule ainsi dans son mémoire final de Ml lorsqu'elle évoque rétrospectivement son cheminement : "J'étais surtout dubitative quant à la possibilité pour l'élève de comprendre la complexité du fait colonial en une ou deux études ciblées ». Doutes que l'on retrouve en effet dans un de ses premiers écrits (janvier de M1) qui montre, à propos des programmes de lycée, sa réticence face la possibilité d'aider les élèves à appréhender la complexité du monde passé en réduisant l'étude à quelques situations : «cf. programmes actuels du lycée: en première, la décolonisation se résume à l'étude de l'Inde et de l'Algérie, donc ne permet pas de comprendre la multiplicité des situations dans le fait colonial français; en terminale, la question de la mémoire ne concerne que l'Algérie $»$. Ce premier travail lui permet de préciser et reformuler cette question en problème didactique, qu'elle compte traiter en deuxième année : « Comment, parallèlement à l'étude de cas, arriver à le [le cas] mettre en perspective en s'interrogeant sur l'ensemble du fait historique, sans refaire un cours linéaire, mais en mettant en avant les distinctions que l'on peut faire de situa- tions différentes de celle de l'étude de cas étudiée? » (mémoire de M1).

\section{La prégnance du problème théorique sur le problème professionnel}

Ces formulations et le processus dans lequel elles s'insèrent sont les indices de questionnements qui touchent essentiellement les idées explicatives que l'on peut rapprocher des généralisations, croyances et valeurs de Kuhn, qui proviennent de trois sources: son expérience, ses lectures théoriques et les textes institutionnels. Pour elle, la question de l'étude de cas pour enseigner l'histoire, c'est le rapport du singulier au général : «Je bloque, je ne sais pas trop ce que je cherche. Peut-être comment on généralise? » (séminaire du mois de mai de M1) ; autrement dit, comment passer d'un ou deux cas réduits, à l'explication scientifique d'un phénomène ou d'un événement historique, c'est-à-dire emprunte d'une certaine généralité? Problème qui révèle en creux l'obstacle que constituent ses représentations de l'enseignement et de l'apprentissage de l'histoire comme transmission d'explications générales, appuyées sur des illustrations à même de les rendre accessibles aux élèves; représentations qui sont en phase avec celles qui dominent dans la profession, par opposition « à l'autre histoire, traditionnelle, énumérative, celle qui se contenterait de décrire au lieu d'expliquer » (Lautier, 1997, p. 134). À l'issue de sa première année, elle parvient donc à mettre formellement en tension ses représentations didactiques du métier (il faut dépasser l'énumération pour faire une « histoire explicative », Lautier, 1997, p. 134-146) qui sont notamment portées par les recommandations du ministère ${ }^{47}$, et les propositions du cadre théorique de la problématisation en histoire scolaire (qui l'incite à faire construire l'explication sur la base d'un cas qui met en discussion les idées explicatives déjà-là des élèves) : si elle ne voit pas à ce stade comment la nécessité de l'histoire explicative peut être remplie par la mise à l'étude d'une situation singulière, elle est cependant convaincue que c'est ce qui est intéressant. Ce qui correspond aux enjeux majoritairement perçus par les enseignants analysés par Lautier : « mais lorsqu'on a délimité le terrain en donnant congé à l'histoire non explicative, il reste à résoudre d'autres ambiguités : dans la recherche d'explications, il faudra éviter l'illusoire causalité linéaire 
sans pour autant tomber dans la reconstruction d'une logique rigoureuse qui exclut toute contingence » (Lautier, 1997, p. 134-135). Le penchant constructiviste assez largement partagé par la profession selon lequel il ne peut suffire d'expliquer pour enseigner s'incarne pour elle dans la possibilité d'une construction du savoir par l'intermédiaire d'un cas accessible aux élèves : ce qui représente une "généralisation » au sens de Kuhn. Mais le résultat auquel elle aboutit en fin de Ml traduit la résistance intrinsèque de l'obstacle que constitue sa difficulté à distinguer le cas de l'exemple et de l'illustration : le modèle inductif qui fait de l'exemple le soutien d'une explication validée par l'autorité professorale l'empêche d'envisager clairement le cas comme « un récit [...] qui souligne les contradictions internes d'une norme ou les contradictions entre deux systèmes normatifs » (Ginzburg, 2010, p. 361). Si le problème didactique de l'étude de cas est posé, le concept lui-même n'est pas approprié au-delà de ses aspects définitoires directement accessibles dans les textes qu'elle a étudiés. Si elle est convaincue que « ces questions de mise en perspective du cas renvoient au va-et-vient entre microhistoire et macro-histoire, à la nécessité, pour que l'étude de cas soit opportune, de changer d'échelle » (mémoire de M1), l'usage qu'elle fait dans son texte des termes micro-histoire / macro-histoire révèle qu'elle tend à réduire l'étude de cas à un travail à échelle réduite qui mériterait ensuite et ailleurs, une généralisation.

\section{Entre théorisation scientifique et théorisation dans la pratique}

Elle apparaît finalement partagée entre deux solutions différemment légitimes parce qu'inscrites dans des registres différents : la théorie issue des cours et des lectures, et d'autre part les représentations qu'elle a du lien entre données et généralisations en histoire, tirées de son expérience scolaire. Son mémoire de fin de première année manifeste cette tension entre deux orientations théoriques de l'ordre des généralisations, croyances et valeurs ${ }^{48}$. Mais ni cette expérience, ni les textes qu'elle utilise, ni ses périodes de stage d'observation ne la conduisent à produire des données spécifiques qu'il s'agirait de confronter à l'une ou l'autre des théorisations en jeu pour définir les relations entre les pratiques et les apprentissages. Elle sélectionne dans ses connaissances des éléments qui lui permettent de confirmer ses idées générales, de manière très implicite, sans les documenter ni les situer $^{49}$. Les échanges du séminaire ne la font pas accéder au second niveau, celui qui mettrait ces généralisations en relation avec des données selon une manière exemplaire, portée par le séminaire et le directeur de mémoire. Le séminaire de Ml ne fonctionne pas comme une communauté scientifique.

La seconde année, au cours de laquelle l'étudiante poursuit son initiation à la recherche mais en parallèle avec une activité d'enseignante à temps partiel (elle a deux classes de collège), elle acquiert - comme deux autres des étudiants du groupe - une position d'expérience, et la possibilité de produire des données qu'elle peut opposer aux autres; le séminaire change alors de nature.

\section{Séminaire, communauté de recherche et exemple exemplaire}

On observe cette modification de position dans le séminaire par le biais des échanges avec son directeur dans lesquels percent des mises en tension entre la logique didactique de recherche et la logique d'enseignement. En tout début de M2, elle lui précise ainsi, avant une réunion du séminaire prévue quelques jours plus tard, qu'elle «pense particulièrement insister non sur "l'étude de cas" en histoire, mais sur la mise en perspective de l'étude ciblée, conformément à ce qui est demandé dans les programmes » (mail, septembre du M2). Cette dernière précision explicite en direction de son directeur le fait qu'elle fait des choix qui se décalent par rapport à ses conseils (transmis auparavant sous la forme d'un bilan du $\mathrm{Ml}$, et qui la guide fortement vers l'étude de cas en histoire), et que ces choix sont légitimés par sa position d'enseignante, soumise à une autre autorité (les programmes).

\section{Le problème didactique réinvesti par la production de données}

Sur cette base nouvelle, son premier texte de M2 (octobre) montre une reprise du problème de la généralisation sur la base de la préparation et la mise en œuvre d'une séquence : le problème didactique précédemment posé est mis à l'épreuve de son travail de classe, c'est-à-dire de la production de données à 
partir de séquences construites et analysées dans ce but. À plusieurs occasions au cours de l'année, elle exprime en séminaire et dans ses textes ses difficultés en insistant sur le hiatus entre ses contraintes de professeur (les résistances des élèves, les contraintes institutionnelles, etc.) et ses idées de didacticienne (contraintes intellectuelles construites dans les cours et les séminaires). Ainsi, à son directeur qui évoque l'idée que le processus auquel elle aboutit est sans fin (des essais à l'analyse, à un nouvel essai, et ainsi de suite), elle répond que « le problème c'est que cette année, et c'est là où je ne suis pas du tout en accord avec la recherche, c'est que je veux faire le cours parfait. Vous voyez bien, mais bon c'est normal [...] mais du coup on s'en moque complètement en recherche, mais du coup c'est comme s'il fallait que je sois coupée en deux, et je n'y arrive pas » (séminaire, mai du M2). La réalité de l'enseignement semble devenir le support de l'explicitation - qui était quasi-inexistante en Ml - de ses propres idées explicatives, de son choix entre les recommandations institutionnelles et les idées de ses lectures didactiques: ses solutions didactiques de professeur ne se superposent pas à ses convictions d'apprentie didacticienne, et cela devient dominant dans ses interventions et ses textes (en particulier un texte d'avril du M2 présente en plus de trois pages la succession des problèmes sur lesquels elle «bute » depuis le début de cette deuxième année; ce qu'elle reprend de manière détaillée lors du séminaire suivant, en mai).

Ce changement de position dans le séminaire ne permet cependant de rendre compte du processus en jeu en M2 que de manière superficielle parce que sans lien explicatif avec ses représentations didactiques en jeu à travers son sujet. Il est plus pertinent de souligner que le séminaire devient le lieu d'une discussion non plus sur l'expérience en général, mais sur des situations d'enseignement effectives. Le directeur de mémoire incite ainsi systématiquement à la production de corpus et, sur cette base, à la construction de données spécifiques, singulières et situées, comme l'illustre l'extrait d'un mail d'octobre de l'année de M2, en réponse à un texte proposé par l'étudiante: « Mais vous dites cela dans la suite de votre texte; arrive plus loin votre premier corpus (sur le jihad) qui me semble très pertinent à ce stade de l'étude. Il faut le développer comme vous le faites pour faire avancer votre réflexion : voir mes commentaires dans le texte».

Ce type d'intervention renvoie à l'organisation systématique des cinq séminaires de l'année de M2.
D'une durée de trois heures, ils se structurent autour des textes envoyés au préalable par les étudiants, qu'ils présentent et soumettent à la discussion du groupe. Pour les étudiants ayant en charge des classes toute l'année (trois sur les cinq), le directeur oriente initialement ces discussions vers la description des séquences qui servent de support aux écrits. Leur position d'étudiants-professeurs leur permet de développer leurs descriptions du point de vue des contraintes de la pratique ${ }^{50}$. Sur cette base, le directeur dirige les échanges vers la mise en relation de ces contraintes avec les apprentissages, ce qui met au cœur de la discussion les enjeux d'interprétation des productions d'élèves (leurs discours, leurs écrits) pour discuter la relation entre pratiques d'enseignement et apprentissage selon les problématiques en jeu ${ }^{51}$.

\section{La production de données favorise la reconstruction du problème didactique}

Ce qui amène à caractériser le modèle (qui se veut exemplaire) de construction et de résolution de problème didactique que met en œuvre cette organisation du séminaire : par contraste avec l'année de M1 et l'habitude professionnelle, l'activité du séminaire réclame la production de données, notamment sur les effets d'apprentissage des pratiques de classe, en relation directe avec les questionnements théoriques. Les données produites sont donc ad hoc: en tension avec les discussions engagées dans le séminaire (et plus directement dans le dialogue écrit avec le directeur de mémoire).

On le constate au fil des évocations du mémoire lui-même dans lequel les données sont insérées systématiquement dans la progression du problème didactique. On y distingue assez nettement les détours qu'elle suit pour concilier son ambition théorique sur l'étude de cas (par opposition à l'idée de « mise en perspective " des documents d'accompagnement de l'Éducation nationale), et les idées issues non plus seulement de son expérience des relations entre pratiques et apprentissages, mais plus directement des essais analysés empiriquement. Ainsi en est-il de la manière dont une reprise serrée d'une situation de classe - en quatrième sur les Grandes découvertes - lui permet de fonder son analyse sur des données, pour immédiatement la confronter à ce qu'elle comprends " des théories des didacticiens concernant les représentations » (Meknassi, 2014, 
p. 27-28). Ou encore dans le corpus qu'elle constitue sur le XIX ${ }^{\mathrm{e}}$ siècle industriel (ibid., p. 32-33) : elle articule les productions des élèves (qui, par exemple, acceptent aisément la juxtaposition de deux idées : « le progrès technique permet globalement de meilleures conditions de vie car on peut posséder plus de biens; les ouvriers ont des conditions de vie difficiles ») et les relations pratiques / apprentissages selon ses réflexions à ce stade, par contraste avec l'étude de cas chez les historiens, pour aboutir à distinguer entre opinions et savoirs historiques : "Dire que les conditions de travail des ouvriers sont difficiles renvoie à une réalité du présent, à une certaine "vérité" qui n'est finalement pas le fruit d'un raisonnement. Étudier le cas Lucie Baud n'a pas vraiment permis aux élèves que cette représentation, qui est une opinion, devienne un savoir, c'est-à-dire un phénomène historique que l'on peut expliquer $»$. Et de la même manière (Meknassi, 2014, p. 33) sur un corpus de séquence sur les conquêtes coloniales.

\section{Le dépassement de ses représentations didactiques}

Par le truchement de ces intrications entre corpus, interprétations des corpus et idées explicatives, confrontés collectivement, elle remet en question le concept d'étude de cas tel qu'elle l'avait appréhendé en Ml. En substance, son raisonnement suit le cheminement suivant. Son analyse historiographique et épistémologique fait de l'étude de cas le processus de mise en discussion de l'état dominant du savoir dans la communauté historienne, mais il n'a pas d'équivalent dans le cadre scolaire. Pour l'enseignante quelle est, les élèves ne partagent pas un savoir historique antérieur suffisamment consistant pour endosser ce rôle. Pour elle, confortée par son expérience d'enseignante, la transposition de l'étude de cas bute sur l'organisation institutionnelle radicalement différente des deux contextes (la classe, le séminaire de recherche) : à plusieurs reprises elle dit et écrit que « les élèves ne sont pas des historiens ». C'est par ce processus qu'elle repense l'étude de cas : ses constats d'enseignante l'obligent à revenir sur ses lectures théoriques pour pouvoir défendre une position au sein du séminaire. Elle explore finalement une hypothèse de solution à ce problème : si les élèves sont trop ignorants sur le plan historique pour avoir des représentations à mettre en discussion, en revanche, sur le plan moral, ils ont toujours des choses à dire : «Chez l'historien, il y a un cas si c'est opposé aux connaissances qu'il a sur un phénomène. L'élève, là où il pourrait y avoir un cas c'est parce que ça s'oppose à ses valeurs [...]. Mais du coup, ça n'a rien à voir avec une démarche scientifique. " (texte, mai du M2). Solution qui s'entend comme une ouverture mais qui présente la faiblesse de ne pas se conformer à la visée scientifique incontournable dans l'approche à défendre en séminaire, notamment face au directeur de recherche. Pour elle, cette piste conduit vers le « jugement moral »: « En fait se dire oui c'est [l'esclavage] mal, bien oui d'accord c'est mal et puis après [...] enfin je trouve que ça ne peut pas vraiment nous servir » (ibid.). Cependant, retraçant son cheminement de recherche, elle évoque le processus d'aller-retour entre écriture d'analyse et conception de séquences qu'elle a enclenché, et qui l'a conduite à reformuler le problème sur la base de cette « mauvaise » solution. Puisqu'ils n'auraient pas de représentations sur tel ou tel point du programme (impossibilité de faire comme chez les historiens), et que les idées explicatives morales ne sont pas de l'ordre du savoir historique (solution rejetée), il lui faut prendre leurs représentations autrement :

«- On nous demande d'étudier la Guerre d'Algérie [...] pour, en quoi elle peut illustrer les autres conquêtes [...]. Finalement, une autre manière d'aborder le cas, puisque là par exemple si je prends le cas de la Guerre d'Algérie, forcément, si je cherche à me dire comment faire pour que tel point ça n'aille pas dans leurs représentations, en fait, sur la conquête d'Algérie ils n'ont pas vraiment de représentations par exemple, forcément c'est trop pointu déjà, et ils n'ont pas, absolument pas d'avis dessus. - [Directeur de recherche] Oui mais sur le fait de faire la guerre dans un autre pays pour le conquérir. - Voilà, donc c'est ce que je me suis dit. Il faut généraliser, enfin il faut voir plus haut. Et donc je me suis dit peut-être que la solution ce serait, sur un plus long terme, peut-être sur plusieurs séquences, penser à un concept. Et donc c'est là que j'ai parlé pour la guerre, mais pour les sociétés coloniales ce serait pareil [...]. Lidée ce serait de dire, comme une guerre ils savent forcément déjà, ils ont forcément un avis sur ce que c'est une guerre, c'est pas comme... là on ne parle pas de quelque chose d'historique. Une guerre c'est aussi quelque chose d'actuel, voilà. Ils ont forcément des idées sur ce qu'est une guerre et donc, ensuite, je vais leur faire étudier par exemple la conquête en Algérie, 
pour essayer de voir la spécificité de la guerre coloniale, et notamment par exemple le fait de s'en prendre directement au peuple [...], les spécificités par rapport à ce qu'on avait vu que pouvaient être les guerres jusqu'à maintenant. Et là je me suis dit que ça pouvait peutêtre être une bonne approche du cas [...]. Et s'attacher en tant que professeur à pouvoir enrichir la co... enfin la réflexion autour d'un concept, et bien ça pousse les élèves à comparer des choses, et bon enfin je trouve que c'est vraiment au cour du travail du professeur d'histoire vu qu'on n'apprend pas, on n'enseigne pas l'histoire pour l'histoire mais vraiment pour que les élèves soient capables de mettre des choses en parallèle, de comparer » (séminaire, mai du M2).

Le renversement est patent d'abord par l'usage de catégories didactiques qui lui permettent de penser l'obstacle qui la gênait, en termes de relations entre des concepts, idées explicatives générales, et leur usage dans des situations historiques (singulières). Ensuite, le cheminement qu'elle retrace indique à la fois la construction du problème dans le cadre théorique du séminaire, et la résolution du problème professionnel qui dominait depuis qu'elle était également devenue enseignante. D'une certaine manière elle indexe l'intérêt du processus de problématisation didactique qui lui permet de sortir de la dichotomie (incarnée ici par le schéma linéaire qui réclame des bases de contenus avant d'imaginer des activités historiennes), à sa capacité à résoudre le problème professionnel.

\section{DISCUSSION}

Cette étude visait à dégager les conditions d'une sortie de l'inertie de la discipline scolaire dans laquelle s'inscrivent les enseignants d'histoiregéographie, en s'appuyant sur la notion d'inconscient scolaire disciplinaire afin de permettre le développement d'une comparaison entre discipline scolaire et discipline universitaire, ce que nous avons tenté par le biais d'un aspect de cet inconscient, celui de la dichotomie entre savoirs (dimension épistémique) et pratiques pédagogiques (dimension institutionnelle). Mais comme nous sommes nous-mêmes, en tant que chercheur, pris dans cet inconscient scolaire disciplinaire, nous avons choisi d'adosser notre approche didactique (le cadre de la problématisation) qui est susceptible de verser du côté épistémique, à des travaux en histoire sociale des sciences, et notamment le concept d'exemple exemplaire de Kuhn.

Cet adossement de l'analyse didactique à l'histoire sociale des sciences gagne sans doute à ce stade à être conceptualisé par l'intermédiaire de la notion d'interdisciplinarité restreinte, au sens de Lepetit (1990). Une des conditions de possibilité de la « pratique restreinte de l'interdisciplinarité », si l'on veut éviter une interdisciplinarité " diffuse, mal perçue, mal contrôlée », réside dans la prise en compte de la résistance de la discipline d'accueil et des effets de transformation sur les innovations importées : « les échanges interdisciplinaires ne s'opèrent pas de manière aléatoire, ils ne peuvent être compris que par rapport au rôle de subversion ou de légitimation qu'ils jouent dans la discipline d'accueil » (Lepetit, 1990, p. 334). En l'occurrence, l'intérêt de l'insertion du concept kuhnien dans notre étude didactique nous paraît résider dans l'effet de légitimation ${ }^{52}$ qu'il produit sur notre perspective didactique - l'apprentissage par problématisation - en allant contre l'idée d'un regard épistémologique coupé de toute préoccupation socio-historique. Voyons comment opère ce processus d'accueil et d'acculturation dans l'étude du cas de cette étudiante.

La rupture didactique que l'on identifie par le biais des concepts du cadre bachelardien de la problématisation (rupture, obstacle, problème) opère à deux niveaux. Elle concerne l'étudiante et son inconscient scolaire disciplinaire, qui constitue sa grille de lecture des situations d'enseignement : l'idée qu'il faut que les élèves acquièrent d'abord des bases en terme de connaissances avant de pouvoir déployer une activité de mise à distance, est mise en question dans le cas du travail scolaire de concepts comme celui de guerre. Cette rupture didactique pose en particulier la question de l'intérêt formateur de l'initiation à la recherche dans la formation professionnelle ${ }^{53}$. Mais cette rupture didactique nous concerne simultanément comme chercheurs en didactique (de l'histoire) parce qu'elle met en question le savoir stabilisé par des recherches antérieures sur l'inertie de cette dichotomie - c'est-à-dire la résistance de cet inconscient scolaire.

Si le cadre théorique de la problématisation permet d'établir la rupture en identifiant les nécessités didactiques produites par l'étudiante pour passer d'une étude de documents à une étude de cas en classe d'histoire, c'est le concept d'exemple exemplaire qui nous permet de poursuivre l'enquête 
pour rendre raison de ce processus de problématisation en en identifiant les conditions de possibilité au sein d'une discipline scolaire marquée par l'inertie. On peut en effet assimiler le processus de recherche de cette étudiante sur les deux années comme une entrée dans une science normale, au sens de Kuhn : non pas une révolution scientifique qui supposerait un fonctionnement déjà inscrit dans une matrice disciplinaire, mais l'intégration à une matrice. En effet, le processus de production de données (sur l'usage que les élèves font de leurs représentations) qui caractérise le travail du séminaire en deuxième année fait apparaître par contraste, aux yeux de l'étudiante, le fonctionnement habituel inconscient qui ne permet pas cette production contrôlée des données. Cette mise en relief opère un changement de nature des principes qui guident le travail du professeur : ces principes qui cadrent et génèrent l'interprétation (en terme d'apprentissage) des énoncés produits par les élèves, auparavant invisibles, se manifestent dans les échanges oraux et écrits du séminaire, non seulement par une prise de conscience de leur existence (déjà centrale dans le travail de la première année pour différencier principes de l'étude ciblée et principes de l'étude de cas), mais aussi par l'élaboration de nécessités de faire appel à tel ou tel principe. Ces nécessités sur les principes émergent du processus de comparaison qui opère entre l'élaboration de la séquence sur la Guerre d'Algérie (mise en perspective avec d'autres séquences sur le concept de guerre), et la construction des séquences ordinaires au fil de son activité d'enseignante. Par exemple, une étude de cas en classe d'histoire nécessite l'appui sur des concepts généraux qui constituent des modèles utilisables pour différents contextes, et non sur des connaissances factuelles. Or cette comparaison n'est possible que parce que l'exceptionnalité du travail sur un concept n'est pas rejetée comme anomalie : elle est au contraire validée comme exemplaire d'une autre manière de faire par le biais du séminaire (et donc du directeur de mémoire qui le pilote et garantit la légitimité de cette nouvelle manière de faire comme science normale).

On discerne dans cette dialectique de l'exceptionnalité et de l'exemplarité la dimension profondément didactique du concept d'exemple exemplaire de Kuhn déjà évoquée à la suite de Sensevy. Le processus décrit n'est pas celui d'une révolution scientifique qui marquerait un changement de paradigme (au sens de la vulgate) pour l'étudiante, mais plutôt celui d'un accès à une science normale qui se marque par une manière plus scientifique d'observer la classe, par opposition avec une manière professionnelle d'agir dans la classe. La différence entre les deux réside dans l'existence d'une communauté d'accueil - telle que la présente Kuhn : une communauté encadrant des exercices exemplaires de production et de résolution de problèmes - qui est ici portée par le chercheur-directeur de mémoire. L'institution « séminaire ", par le rattachement du directeur à la communauté scientifique, et par son fonctionnement caractérisé par une circulation et une validation des textes spécifiques, établit et rend visibles pour l'étudiante deux manières d'analyser son travail, l'une ordinaire dans la pratique, et l'autre exemplaire dans le séminaire, incarnée par le cas de la séquence sur le concept de guerre.

Ce détour interdisciplinaire par la notion sociologique d'inconscient scolaire et par le concept d'exemple exemplaire issu d'études d'histoire sociale des sciences donne finalement à penser autrement certaines questions didactiques (ici appréhendées par le prisme du cadre théorique de la problématisation), comme celle du lien entre apprentissage (en didactique) et développement (professionnel) chère à Jean-Paul Bernié (Bernié, 2002).

D'une part, le changement de nature des principes en jeu dans l'activité enseignante engendré par la rupture didactique observée peut modifier radicalement le rapport à l'activité. En rendant visible l'écart entre usage de principes en actes et usage différé par un dispositif d'observation, c'est la convergence entre la pratique didactique et la recherche didactique qui est soulignée, et par là la place d'une formation à la recherche dans le développement professionnel qui est posée. Enseigner c'est produire des séquences à partir de l'expérience antérieure et de l'évaluation de l'efficacité de ces dispositifs en terme d'apprentissage, évaluation qui passe par une interprétation plus ou moins contrôlée. Penser ainsi l'enseignement de la discipline c'est alors, pour le chercheur, « pulvériser $»$ la notion de séquence (Bernié, 2002, p. 82).

D'autre part, le dépassement de l'inconscient en jeu dans notre étude pose la question du rapport entre faire problématiser (des élèves, des étudiants) et leur enseigner à problématiser, autrement dit, entre faire apprendre un savoir historique par problématisation, et développer la capacité à problématiser. Comme le souligne Orange (2007, p. 12) à propos de l'observation d'un processus de problématisation 
avéré dans une classe par la production de nécessités mais qui mettent en jeu des principes uniquement utilisés en actes, « si le travail fait dans cette classe est "à la mode des problématisations scientifiques", il n'en est pas une »: le degré de généralité et d'abstraction de ces nécessités reste faible par absence d'articulation explicite aux principes dans lesquels elles prennent sens. Une des conditions de possibilité d'explicitation des principes inconscients qui régissent ces processus mis en lumière par notre étude résiderait dans la production d'exemples exemplaires d'une manière scientifique de faire et de voir, qui a à voir avec la production d'une communauté scientifique, même réduite.

\section{NOTES}

1. Chervel, 1988.

2. «Je peux dire qu'un de mes combats les plus constants, avec Actes de la recherche en sciences sociales notamment, vise à favoriser l'émergence d'une science sociale unifiée, où l'histoire serait une sociologie historique du passé et la sociologie une histoire sociale du présent » (Bourdieu, 1995, p. 111).

3. Sur cette notion, voir notamment Boutier, Passeron et Revel (2006) et Heilbron et Gingras (2015).

4. Fabiani explicite ce point : "d'un côté sont définies les conditions de la reproduction d'un corps de savoirs à travers la fidélité à des exercices; de l'autre l'exigence de progrès scientifique stipule que l'horizon de la recherche ne peut être entièrement défini par le fonctionnement régulier de dispositifs institutionnels».

5. Selon la recension de l'ouvrage par Olivier Reboul, Revue française de pédagogie, 71, avril 1985, p. 71-73.

6 . Autour des travaux de Lee S. Shulman (cf « Those who understand: knowledge growth in teaching ».Educational Researcher, 15, février 1986, p. 4-14) et de son concept de Subject matter knowledge: Hunter McEwan et Barry Bull, "The Pedagogic Nature of Subject Matter Knowledge", American Educational Research Journal, 28, été 1991, p. 316-334; et pour la didactique de l'histoire : Sam Wineburg, "On the Reading of Historical Texts. Notes on the Breach between Scholl and Academy", American Educational Research Journal, 28, automne 1991, p. 495-519; Peter Seixas, "The community of inquiry as a basis for knowledge and learning : the case of history ", American Educational Research Journal, 30, 1993 , p. 305-324.
7. Bruno Benoît, Lettre ouverte à Madame la Ministre de l'Éducation nationale, [en ligne], 23 février 2015, disponible sur: [https://www.aphg.fr/Lettre-ouverte-a-Madamela-ministre-de-l-education-nationale] (page consultée le 21 février 2016).

8. Voir Doussot (2010).

9. Pour Guy Vincent, Bernard Lahire et Daniel Thin, la forme scolaire est caractérisée par « la constitution d'un univers séparé pour l'enfance, l'importance des règles dans l'apprentissage, l'organisation rationnelle du temps, la multiplication et la répétition d'exercices n'ayant d'autre fonction que d'apprendre et d'apprendre selon les règles * (Lahire, Thin \& Vincent, 1994). Du point de vue de ce qu'il appelle une "épistémologie du discours pédagogique ", Philippe Meirieu décrit la manière qu'ont les hommes de faire avec la contradiction majeure de la situation pédagogique (accompagner l'autonomisation) et qui s'articule autour du dire et du faire (Meirieu, 1995). Du point de vue historique, on peut référer au travail de Bruter qui vise à rendre compte des formes de fréquentation du passé qui se succèdent et s'articulent depuis le XVII ${ }^{\mathrm{e}}$ siècle jusqu'aux formes actuelles (Bruter, 1997).

10. Ministère de l'Éducation nationale, « Vade-mecum des capacités en histoire-géographie-éducation civique », [en ligne], novembre 2011, disponible sur : [http://cache. media.eduscol.education.fr/file/Competence_5/45/1/Vademecum_HGEC_Critique_198451.pdf] (page consultée le 4 février 2016).

11. Perspective visible notamment dans les évaluations internationales des systèmes éducatifs comme les enquêtes PISA de l'OCDE : «PISA is unique because it develops tests which are not directly linked to the school curriculum. The tests are designed to assess to what extent students at the end of compulsory education, can apply their knowledge to real-life situations and be equipped for full participation in society " "What makes PISA different", [en ligne], disponible sur: [http://www.oecd.org/pisa/aboutpisa/], page consultée le 10 février 2016).

12. Dans l'approche de Bourdieu, la domination est un des effets du développement de dispositions qui font accepter - par violence symbolique - les structures institutionnelles dont les agents font l'expérience.

13. «Le socle s'organise en sept grandes compétences: chacune est composée de connaissances essentielles, de capacités à les utiliser et d'attitudes indispensables tout au long de sa vie », ministère de l'Éducation nationale, « Le socle commun de connaissances et de compétences », [en ligne], disponible sur : [http://www.education.gouv.fr/ cid2770/le-socle-commun-de-connaissances-et-de-competences.html] (page consultée le 10 février 2016). 
14. Cf. infra. Cette perspective est explicite dans les ressources proposées aux enseignants. Voir par exemple: ministère de l'Éducation nationale, « Des démarches pour la mise en œuvre du programme d'histoire ", [en ligne], 30 juin 2010, disponible sur: [http://cache.media.eduscol.education.fr/file/lycee/76/1/LyceeGT_Ressources_ HGEC_2_Hist_01_Demarches_hist_148761.pdf] (page consultée le 10 février 2016).

15. C'est ce que Nicole Tutiaux-Guillon synthétise sous la notion de "paradigme pédagogique positiviste », indiquant ainsi l'ancrage des activités de l'histoire scolaire dans une époque historiographique largement révolue et au moins partiellement déconsidérée (Tutiaux-Guillon, 2008). Pour une analyse empirique détaillée et récente, voir Cariou, 2013.

16. «J'ai déjà fait allusion à un modèle de l'activité d'enseignement dans lequel enseigner est le produit d'une division du travail, entre les chercheurs-producteurs [scholar-producers] et les enseignants-transmetteurs [teacher-transmitters]. Le troisième groupe dans le modèle - les élèves - sont conçus comme consommateurs [consumers]) » (Levisohn, 2008).

17. «L'inconscient scolaire est un arbitraire historique qui, du fait qu'il a été incorporé et, par là, naturalisé, échappe aux prises de la conscience - notamment parce qu'il porte à appréhender comme naturelles les structures dont il est le produit. Étant peu à peu devenu consubstantiel à l'activité intellectuelle, il ne peut être saisi que dans ses manifestations ou ses effets objectifs, c'est-à-dire par l'enquête empirique, historique ou sociologique, fonctionnant comme expérience épistémologique » (Bourdieu, 2000b, p. 3).

18. Une illustration en est offert par un texte de V. Duclert, alors récemment nommé président du jury du CAPES d'histoire-géographie : « un enseignant qui participe à la fabrique des savoirs renforce par son expérience de recherche sa liberté de transmettre autant que sa propre assurance et son autorité. Les établissements gagnent à bénéficier de l'influence de ces enseignants également chercheurs, comme ceux qui contribuent aux services éducatifs d'archives » ( $P$ Profs d'histoire et chercheurs », [en ligne], La vie des idées, 3 septembre 2013, [http://www.laviedesidees.fr/Profs-d-histoire-et-historiens.html] (page consultée le 8 décembre 2015).

19. Vincent Duclert, « Rapport de jury capes externe histoire-géographie, session 2014 rénovée ", [en ligne], disponible sur : [http://cache.media.education.gouv.fr/file/ capes_ext/56/9/RAPPORT_JURY_CAPES_HIST_-_GEO_-SESSION_2014_Renove_346569.pdf] (page consultée le 4 février 2016).
20. Dossier « Difficile enseignement de l'histoire », Le débat, $\mathrm{n}^{\circ} 175$, mai 2013, et dossier " La culture du passé », Le débat, $\mathrm{n}^{\circ} 177$, novembre 2013.

21. Selon les éléments d'autobiographie scientifique que propose Kuhn dans sa préface à La tension essentielle, la circularité en question naît de l'impossibilité d'expliquer la science normale qu'il étudie historiquement par un consensus explicite entre chercheurs d'une même communauté ou par une croyance, un paradigme partagé, sans lier les deux : «À cette époque, je voyais la science normale comme résultant d'un consensus entre les membres d'une communauté scientifique. Mais je rencontrais des difficultés lorsque j'essayais de spécifier le consensus en énumérant les éléments auxquels les membres d'une communauté donnée étaient censés adhérer. Pour rendre compte de la manière dont ils menaient leur recherche $[. .$.$] je devais$ leur supposer un accord commun dans leurs définitions des caractéristiques de termes quasi théoriques comme « force » et « masse » [...]. Mais mon expérience, tant scientifique que d'historien, m'avait fait entrevoir que ces définitions étaient rarement enseignées et que les tentatives de les formuler débouchaient souvent sur de profonds désaccords. Apparemment, ce consensus que je cherchais n'existait pas. Pourtant, je ne vis aucun moyen d'écrire le chapitre sur la science normale sans y faire appel $»$ (Kuhn, 1990, p. 23).

22. Une récente tribune a été publiée, signée par de nombreux professeurs de l'enseignement scolaire (et accompagnée d'une pétition signée par des chercheurs de renom) qui exprime les difficultés à exercer simultanément le métier d'enseignant et le travail de recherche ( "L'Éducation nationale méprise-t-elle ses chercheurs? », [en ligne], Libération.fr, 22 octobre 2014, [http://www. liberation.fr/societe/2014/10/22/l-education-nationalemeprise-t-elle-ses-chercheurs_1127256], page consultée le 8 décembre 2015).

23. Voir en particulier les positions extrêmes des sciences de l'éducation objectivées dans les graphiques mettant en relation les origines sociales et scolaires des étudiants, et les disciplines universitaires.

24. Rejet déjà analysé en 1985, à l'aube du développement scientifique de la didactique de l'histoire par Crubellier (1985); plus récemment, au-delà du cas de l'histoire, par Chevallard (2007).

25. Le rapprochement entre didactique et sociologie des sciences repose notamment sur une proximité : elles constituent toutes deux un lieu de dévoilement de ce qui ne se dévoile pas dans le fonctionnement quotidien de la pratique académique, mais qui en constitue un enjeu; cf. Bourdieu (1975). 
26. Dont les choix, bien que discutables du point de vue d'un didacticien - qu'est, par exemple, le « paradigme de la "transposition didactique", et en quoi serait-il à l'origine des travaux de didactique de l'histoire dans le cadre de la discipline scolaire? - renvoient à la possibilité d'une rencontre entre didacticiens et historiens par l'épistémologie (Delacroix, 2015, p. 200-203).

27. Que Deleplace (2016) analyse comme « moment épistémologique » (p. 53) et qu'il identifie à la perspective dominante de l'épreuve sur dossier depuis le milieu des années 2000 jusqu'à la récente réforme.

28. Il note, à propos des textes de cadrage du nouveau capes d'histoire-géographie, que « les incertitudes du vocabulaire, qui établissent une équivalence floue entre le pédagogique, le didactique et le professionnel, ajoutent encore à cette impression d'un difficile compromis » (Delacroix, 2015, p. 196).

29. Voir Doussot (2017).

30. Voir sur ce point notamment l'analyse de la Méditerranée de Braudel par Noiriel (2002).

31. Ibid. ( exemplary exercise »).

32. Comme le formule l'auteur dans un article plus théorique (Seixas, 1993, p. 316).

33. La philosophie du problème de Bachelard s'efforce « de souder épistémologie et pédagogie » (Fabre, 2009, p. 14).

34. «Il faudrait donc pousser les élèves, pris en groupe, à la conscience d'une raison de groupe, autrement dit à l'instinct d'objectivité sociale, instinct qu'on méconnaît pour développer de préférence l'instinct contraire d'originalité, sans prendre garde au caractère truqué de cette originalité apprise dans les disciplines littéraires. Autrement dit, pour que la science objective soit pleinement éducatrice, il faudrait que son enseignement fût socialement actif » (Bachelard, 1938, p. 244).

35. Comme elle fait écho à la formulation de Wittgenstein : « Pour établir une pratique, les règles ne suffisent pas; on a également besoin d'exemples. Nos règles laissent ouvertes des portes de sortie, et la pratique doit parler pour ellemême » (Wittgenstein, 2006, p. 52).

36. Pour lui, la notion d'exemple exemplaire « montre l'aspect fondamentalement didactique de la notion de paradigme »(Sensevy, 2011, p. 208).

37. «L'étudiant apprend à voir, avec ou sans l'aide de son instructeur, que son problème ressemble à un problème qu'il a déjà rencontré. Une fois qu'il a vu la ressemblance et saisi l'analogie entre deux ou plusieurs problèmes distincts, il peut établir une relation entre les symboles et la rattacher à la nature d'une manière qui s'est déjà révélée efficace. L'aptitude qui en résulte de voir qu'un certain nombre de situations se ressemblent [...] est, me semble-t-il, l'essen- tiel de ce qu'acquiert un étudiant en faisant des problèmes à titre d'exemples, soit avec un crayon et du papier, soit dans un laboratoire bien conçu. [...] Dans l'intervalle, il a assimilé une manière de voir autorisée par le groupe et éprouvée par le temps » (Kuhn, 1983, p. 257).

38. Notamment tels qu'ils sont développés à partir de la postface de la réédition de son ouvrage de 1962 (La structure des révolutions scientifiques), publiée en 1969, et du chapitre « Second thoughts on paradigms » publié en 1977 (Kuhn, 1990, chap. XII).

39. Au sens de skholé, de temps d'étude hors de l'urgence de la pratique.

40. Pour une description de ces masters, voir le site du ministère : [http://www.enseignementsup-recherche. gouv.fr/cid67107/tout-comprendre-du-master-meef-etdes-concours-enseignant-la-f.a.q.html] (page consultée le 21 février 2016).

41. Dans la configuration institutionnelle actuelle issue de la réforme dite « Peillon » de la formation des enseignants (2013), les masters des métiers de l'enseignement, de l'éducation et de la formation (MEEF) se déclinent en une mention spécifique aux enseignants disciplinaires du second degré, elle-même divisée en parcours disciplinaires (ou bi-disciplinaires comme dans le cas de l'histoire-géographie).

42. Caractère problématique qui se lit dans le rapport de l'inspection générale sur la mise en place des Écoles supérieures du professorat et de l'éducation (ESPE) qui souligne que les étudiants-stagiaires interviewés ont du mal à situer dans la formation « la dimension recherche des mémoires [...] qui se traduit par [...] une déconnexion de la pratique professionnelle », ministère de l'Éducation nationale, de l'enseignement supérieur et de la recherche, Inspection Générale, « La mise en place des écoles supérieures du professorat et de l'éducation », septembre 2014, p. 135. Constat empirique et institutionnel qui fait écho aux analyses déjà anciennes de Gingras, sur le cas des sciences de l'éducation: «dans ce contexte, les professionnels formés à l'université sont dans une situation paradoxale : ils s'attendent à recevoir une formation adaptée aux problèmes pratiques qu'ils devront résoudre et reçoivent souvent une formation axée sur ceux de la discipline » (Gingras, 1991, p. 53).

43. Justification qui peine à relier les différentes composantes de la formation en se fondant, comme dans le cadre de ce rapport de l'inspection générale, sur des catégories peu favorables, qui séparent d'emblée la recherche du reste : «Le mémoire [...] est parfois orienté uniquement recherche et déconnecté du terrain », ibid., p. 80. 
44. Le directeur de recherche est l'auteur du présent article. Le corpus de données sur lequel repose ce cas est constitué des écrits échangés entre le directeur et l'étudiante (dont les mémoires de M1 et M2) sous la forme de mails et de textes annotés, et des prises de notes du directeur à l'occasion des séminaires, sur les deux années de formation. En outre, le mémoire de l'étudiante sur laquelle se centre cette étude de cas est publié sur le site Dumas du CNRS (Meknassi, 2014), tout comme celui d'une autre participante au séminaire (Blondet, 2014).

45. Une partie de ces cours sont assurés par le directeur de recherche.

46. Voir Doussot (2012), et Doussot et Vézier (2014) qui présente une enquête didactique menée dans des classes, et qui est le support d'une partie des cours suivis par cette étudiante.

47. Une partie importante de son mémoire et du travail d'écriture et de discussion qui y mène consistent à interpréter ces recommandations en fonction des propositions épistémologiques et historiographiques concernant l'étude de cas.

48. En voici quelques recensions dans son mémoire de M1 : le rapport entre étude de documents et critique : «L'étude d'un cas semble reprendre les principes de la traditionnelle étude de documents, répandue depuis des années dans l'enseignement de l'histoire, à laquelle on ajoute une critique de ces documents » (p. 12) ; l'idée de déconstruction : «ne serait-ce que pour déconstruire l'idée qu'il en a » (p. 14); l'idée de critique documentaire : « Il importe donc de faire une place importante à l'analyse critique des documents avec les élèves et de leur apprendre à distinguer une source d'un document de synthèse » (p. 15) ; le rapport situation / contexte : « l'étude de cas nécessite une certaine connaissance du contexte » (p. 15); le rapport singulier / complexité : «Choisir comme étude de cas un évènement exceptionnel n'est pas contre-productif et permet quand même d'aboutir à un élargissement du moment que la complexité du phénomène est bien prise en compte » (p. 16).

49. Comme ici, en conclusion : "J'étais surtout dubitative quant à la possibilité pour l'élève de comprendre la complexité du fait colonial en une ou deux études ciblées » (M1, p. 17).

50. Ainsi, à un commentaire du directeur à une autre étudiante du séminaire, elle réagit solidairement en tant qu'enseignante: « mais je pense que ce qui peut nous freiner c'est que les élèves... enfin là où on pourrait dire que leur interprétation est fausse, c'est que leurs postulats vont être faux au départ et que ça peut nous gêner. Enfin je pense que c'est ça qui est compliqué, pour Claire dans son débat... C'est compliqué de se dire on va les laisser... » (séminaire, mois de mai du M2).

51. Comme par exemple ce commentaire du directeur sur son texte d'avril du M2 : « Il faudra expliciter en quoi cet exemple illustre ce que vous dites dans le paragraphe précédent : ce qui va vous obliger à revenir sur ce que sont les types de représentations en jeu (voir mes commentaires précédents) », à propos de cette proposition de l'étudiante : « Il serait alors facile de faire émerger cette représentation en confrontant une photo fin $\mathrm{XIX}^{\mathrm{e}}$ et une photo du $\mathrm{XXI}^{\mathrm{e}}$ siècle».

52. Chez Lepetit cette légitimation n'est pas une marque de dépendance - ici de la didactique envers l'histoire des sciences ou l'épistémologie - parce qu'elle s'inscrit dans un processus par lequel « une discipline ne s'expose jamais passivement aux influences externes » (Lepetit, 1990, p. 334).

53. Ce que nous proposons dans un texte qui analyse sous cet angle le même cas (texte soumis pour publication pour un dossier de la revue Recherches en éducation).

\section{RÉFÉRENCES}

Bachelard, G. (1938). La formation de l'esprit scientifique. Paris : Vrin.

Bachelard, G. (1949). Le rationalisme appliqué. Paris : Presses universitaires de France.

Bazin, J. (2008). Des clous dans la Joconde : l'anthropologie autrement. Toulouse : Anacharsis.

Bernié, J.-P. (2002). Lapproche des pratiques langagières scolaires à travers la notion de « communauté discursive » : un apport à la didactique comparée? Revue française de pédagogie, 141, 77-88.

Blondet, C. (2014). Construire un savoir et entrer dans la pratique historienne en débattant en classe. Repéré à : [http://dumas.ccsd.cnrs.fr/dumas-01222734/ document].

Bourdieu, P. (1975). La spécificité du champ scientifique et les conditions sociales du progrès de la raison. Sociologie et sociétés, 7(1), 91-118.

Bourdieu, P. (1995). Sur les rapports entre la sociologie et l'histoire en Allemagne et en France. Actes de la recherche en sciences sociales, 106, 108-122.

Bourdieu, P. (2000a). Esquisse d'une théorie de la pratique. Paris : Seuil.

Bourdieu, P. (2000b). Linconscient d'école. Actes de la recherche en sciences sociales, 135, 3-5.

Bourdon, E., Chevalier, D., De Cock, L., Delacroix, C., Garcia, P., \& Leduc, J. (2015). Le CAPES d'histoire et de géographie. Vingtième Siècle. Revue d'histoire, 126, 163-176. 
Boutier, J., Passeron, J.-C., \& Revel, J. (2006). Qu'est-ce qu'une discipline? Paris : Éditions EHESS. Repéré à : [http://cat.inist.fr/?aModele=afficheN\&cps $\mathrm{idt}=18501504]$.

Braxmeyer, N. (2007). Les pratiques d'enseignement en histoire, géographie et éducation civique au collège. Éducation et Formations, 76, 95-104.

Bruter, A. (1997). L'histoire enseignée au Grand Siècle. Naissance d'une pédagogie. Paris : Belin.

Bruter, A. (2007). Pédagogies de l'histoire. Histoire de l'éducation, 114, 5-23.

Cariou, D. (2013). Les déséquilibres entre contrat et milieu dans une séance d'histoire à l'école primaire. Une étude exploratoire. Éducation E Didactique, 7(1), 9-32.

Chervel, A. (1988). Lhistoire des disciplines scolaires : réflexions sur un domaine de recherche. Histoire de l'éducation, 38, 59-119.

Chevallard, Y. (2007). Éducation \& didactique : une tension essentielle. Éducation et Didactique, 1(1), 9-28.

Crubellier, M. (1985). Enseigner l'histoire : analyse historique d'un malaise. Histoire de l'éducation, 26, 39-60.

De Cock, L. (2015). L'histoire scolaire, une matière indisciplinée. Annales. Histoire, sciences sociales, 70(1), 179-190.

Delacroix, C. (2015). Un tournant pédagogique dans la formation des enseignants. Les cas du Capes d'histoiregéographie. Annales. Histoire, sciences sociales, 70(1), 191-204.

Deleplace, M. (2016). La place de la didactique dans les concours de recrutement. Retour sur l'expérience de l'ESD du CAPES d'histoire-géographie. Dans S. Lalagüe-Dulac, P. Legris \& C. Mercier (dir.), Didactique et histoire. Des synergies complexes (p. 43-56). Rennes: Presses universitaires de Rennes.

Dewey, J. (1990). Démocratie et éducation. France : Armand Colin.

Doussot, S. (2010). Pratiques de savoir en classe et chez les historiens : une étude de cas au collège. Revue française de pédagogie, 173, 85-104.

Doussot, S. (2012). Le cas Menocchio et la construction en histoire. Le cartable de Clio, 12, 111-125.

Doussot, S. (2017). Recherches historiques, recherches didactiques et formation à l'enseignement de l'histoire. Vingtième Siècle. Revue d'histoire, 133, 129-143.

Doussot, S., \& Vézier, A. (2014). Des savoirs comme pratiques de problématisation : une approche sociocognitive en didactique de l'histoire. Éducation $\mathcal{E}$ Didactique, 8(3), 111-139.

Durkheim, É. (1938). Lévolution pédagogique en France. France : Presses universitaires de France.

Fabiani, J.-L. (2006). À quoi sert la notion de discipline? Dans J. Boutier, J.-C. Passeron \& J. Revel, Qu'est-ce qu'une discipline (p. 11-34). Paris : Éditions EHESS. Repéré à : [http://editions.ehess.fr/uploads/tx_ editionsehess/2-7132-2103-X_REsumesE5.pdf].

Fabre, M. (2009). Philosophie et pédagogie du problème. Paris : J. Vrin.
Fournier, M., Gingras, Y., \& Mathurin, C. (1988). Lévaluation par les pairs et la définition légitime de la recherche universitaire. Actes de la recherche en sciences sociales, 74, 47-54.

Garcia, P., \& Leduc, J. (2003). L'enseignement de l'histoire en France de l'Ancien Régime à nos jours. Paris : Armand Colin.

Ginzburg, C. (2010). Mythes, emblèmes, traces. Morphologie et histoire. (M. Reuff, M. Aymard, C. Paolini \& E. Bonan, Trad., nouvelle édition augmentée). Lagrasse : Verdier.

Girault, B. (2015). De la didactique à l'épistémologie de l'histoire : une réflexivité partagée. Annales. Histoire, sciences sociales, 70(1), 205-214.

Goody, J. (1979). La raison graphique. La domestication de la pensée sauvage. Paris : Minuit.

Heilbron, J., \& Gingras, Y. (2015). La résilience des disciplines. Actes de la recherche en sciences sociales, 210, 4-9.

Johsua, S., \& Lahire, B. (1999). Pour une didactique sociologique. Éducation et sociétés, 4(2), 29-56.

Kuhn, T. S. (1983). La structure des révolutions scientifiques (L. Meyer, Trad.). Paris : Flammarion.

Kuhn, T. S. (1990). La Tension essentielle tradition et changement dans les sciences. (M. Biezunski, P. Jacob, A. Lyotard-May \& G. Voyat, Trad.). Paris : Gallimard.

Lahire, B., Thin, D., \& Vincent, G. (1994). Sur l'histoire et la théorie de la forme scolaire. Dans G. Vincent (dir.), L'éducation prisonnière de la forme scolaire? Scolarisation et socialisation dans les sociétés industrielles (p. 11-48). Lyon: Presses universitaires de Lyon.

Lautier, N. (1997). À la rencontre de l'histoire. Villeneuved'Ascq : Presses universitaires du Septentrion.

Lautier, N., \& Allieu-Mary, N. (2008). La didactique de l'histoire. Revue française de pédagogie. Recherches en éducation, 162, 95-131.

Lepetit, B. (1990). Propositions pour une pratique restreinte de l'interdisciplinarité. Revue de synthèse, 111(3), 331-338.

Levisohn, J. A. (2008). Building bridges to overcome breaches: school and academy, content and pedagogy, scholarship and teaching. South Atlantic Philosophy of Education Society Yearbook, 14-24.

Meirieu, P. (1995). La pédagogie entre le dire et le faire. Paris : ESF.

Meknassi, É. E. (2014). Didactique de l'étude de cas en histoire. Repéré à : [https://dumas.ccsd.cnrs.fr/ dumas-01222541/document].

Milner, J.-C. (1984). De l'école. Paris : Seuil.

Noiriel, G. (2002). Comment on récrit l'histoire. Les usages du temps dans les Écrits sur l'histoire de Frenand Braudel. Revue d'histoire du XIX ${ }^{e}$ siècle, 25, 57-81.

Orange, C. (2006). Analyse de pratiques et formation des enseignants. Recherche et formation, 51, 119-131.

Orange, C. (2007). Spécificité de la problématisation scientifique : le travail d'abstraction et de généralisation. Recherches en éducation, 3, 8-13.

Orange, C. (2012). Enseigner les sciences : problèmes, débats et savoirs scientifiques en classe. Bruxelles: De Boeck. 
Pace, D. (2004). The amateur in the operating room : History and the scholarship of teaching and learning. The American Historical Review, 109(4), 1171-1192.

Renisio, Y. (2015). Lorigine sociale des disciplines. Actes de la recherche en sciences sociales, 210, 10-27.

Rey, B. (2011). Situations et savoirs dans la pratique de classe. Recherches en éducation, 12, 35-49.

Rey, B., Carette, V., Defrance, A., \& Kahn, S. (2006). Les compétences à l'école: apprentissage et évaluation. Bruxelles : De Boeck.

Seixas, P. (1993). The community of inquiry as a basis for knowledge and learning: The case of history. American Educational Research Journal, 30(2), 305-324.

Seixas, P. (1999). Beyond'content'and'pedagogy': In search of a way to talk about history education. Journal of curriculum studies, 31(3), 317-337.

Sensevy, G. (2011). Le sens du savoir : éléments pour une théorie de l'action conjointe en didactique. Bruxelles: De Boeck.

Tutiaux-Guillon, N. (2008). Interpréter la stabilité d'une discipline scolaire : l'histoire-géographie dans le secondaire français. Dans F. Audigier \& N. Tutiaux-Guillon (dir.), Compétences et contenus. Les curriculums en question (p. 117-146). Bruxelles: De Boeck.

Waquet, F. (2000). Parler. La disparition historiographique de la parole magistrale. Actes de la recherche en sciences sociales, 135, 39-47.

Wittgenstein, L. (2006). De la certitude (D. MoyalSharrock, Trad.). Paris : Gallimard. 